\title{
Clinging Onto the Cliff: A Model of Financial Misconduct
}

July 30, 2021

\begin{abstract}
We propose a novel model of financial misconduct. In line with empirical evidence thereon, our model interprets white-collar crime as gambles with skewed payoffs, as opposed to Becker's analysis of criminal activity that postulates positive expected payoffs associated to crime. In our model, criminal motives arise as optimal responses to a "tunnel vision" that engross firm managers, whereby the intense pressure to attain the focused goal triggers strong demand for negatively skewed bets in the form of crime. The key mechanism is consistent with the notion of a "slippery slope to crime" that is finding growing support in the literature as well as in practitioner accounts. Comparative static analyses on the model reveal several empirical implications - for example, a "pecking order of crime" indicating that serious infringements will only follow the depletion of the more preferred (and possibly prevalent) option of milder incursions of law, e.g., minor violations of financial reporting standards - many of which find empirical support in the literature.
\end{abstract}




\section{Introduction}

What motivates or triggers white-collar crimes, in particular, financial misconduct? This is a topic of much interest to researchers and the general public alike. An established framework in this regard involves a cost-benefit analysis of crime, in the spirit of Becker (1968). A somewhat different approach that finds growing support in the literature -with ample support from practitioner's accounts as well- is a pressure-based story; namely that an intense pressure to attain a salient target triggers financial misconduct. A vivid example of this target-induced pressure is given by Soltes (2016, p199):

The Wall Street number was pure. It was somebody else independent of me saying, "Stephen, this is what you need to aim for this quarter." I would judge my success on the ability to make that number. If we achieved that number, it was an endorsement that we were doing the right things. If we missed that number, then it was a reflection that we hadn't performed as well as we should have. My goal was just to get to or over that number -and if I did that, I succeeded. (Stephen Richards, Global Head of Sales at Computer Associates)

Clearly, the executive here is pressured to achieve a focused goal, -the forecast made by Wall Street analysts- the attainment of which would presumably deliver a pulse of utility as the pressure dissipates. As is evident in the executive's narrative, the pulse is concentrated around one particular point, and the incentive is to beat that number, even if by a very narrow margin. Thus, the intensity of focus creates a jump, or dis-continuity on the manager's notional "utility function" around the target point.

Furthermore, the following statement from an AAER filing (No. 2232, for Coca-Cola, SEC 2005) illustrates how these dis-continuities can precipitate white-collar crime:

From 1990 to 1996, Coca-Cola consistently met or exceeded earnings expectations while achieving a compound annual earnings per share growth of $18.3 \% \ldots$ In the mid-1990s, Coca-Cola began experiencing increased competition and more difficult economic environments. Nevertheless, Coca-Cola maintained between 1996-1999 that it expected its earnings per share to continue to grow between 15-20\% annually. At or near the end of each reporting period between 1997 and 1999, Coca-Cola implemented a "channel 
stuffing ${ }^{1}$ " practice in Japan... for the purpose of generating revenue to meet both annual business plan and earnings targets. The income generated by channel stuffing in Japan was the difference between Coca-Cola meeting or missing analysts' consensus or modified consensus earnings estimates for 8 out of 12 quarters from 1997 through 1999.

Evidently, Coca-Cola in this example resorted to the illegal practice of "channel stuffing" in order to meet an expectation (earnings benchmarks) that was formed during a boom, which subsequently became unattainable as business conditions deteriorated, generating a pressure upon the management. Just as in the example given by Soltes (2016), the pressure to meet the expectation induced a dis-continuity, which in turn triggered an incentive to misbehave, one that eventually led to an SEC investigation.

The episodes we introduced represent a prevalence which, undeservedly, is not matched by any existing model. The goal of this paper is to present a model that formalizes and develops the intuition illustrated by the above; namely that the pressure-based dis-continuity generates an incentive to misbehave. Various potential sources of managerial pressure have been discussed extensively in the literature, for example, in the form of the stock market punishing a missed analyst forecast by as little as a single penny (e.g., Jensen 2005 or Bhojraj et al. 2009), a compensation target that must be met to secure a bonus (e.g., Bebchuk et al. 2002 or Burns and Kedia, 2006), internal organizational pressure (e.g., Feng et al., 2011), or simply a managerial hubris/reputation to uphold (e.g., Malmendier and Tate 2005). Whatever the source, we encapsulate the essence of this pressure as a dis-continuous jump in the manager's utility function, and elucidate the key factors and mechanism that lead on to financial misconduct by allowing an optimizing agent to respond to this dis-continuity. The results we obtain resonate with a growing body of research on financial frauds claiming that the events leading up to crime is more complex than a simple execution of cost-benefit analysis. For example, our model speaks to the notion of a "slippery slope" to fraud (Schrand and Zechman, 2012), a situation where the fraud scheme is initially seeded by something rather innocuous -e.g., optimistic bias- but incrementally ensnares the manager into commitment of more serious misconduct. As is also the focus in our model, the process of such entrapment is typically accompanied by a growingly narrow "tunnel vision" (Chu et al., 2019) where the pressure to exceed a target - especially if the target has been beaten consistently before- eventually compels the manager to misbehave.

\footnotetext{
${ }^{1}$ The illegal practise of jamming products into distribution channels to inflate sales.
} 
In our model, crime presents itself as a risky, but attractive temptation to mitigate such managerial pressure. As in our Coca-Cola example, consider a CEO contemplating a "channel stuffing" scheme. If executed successfully, -i.e., without being detected- the manager could deceive the market into believing that the company is in better shape than it really is. If detected however, the company will most likely find itself in a worse shape than the "truthful counterfactual", as the company faces prosecution and its consequences. We thus model crime as a risky enterprise where the outcome is either "good" if undetected, or "bad" if detected, i.e., a gamble of binomial outcomes with a "success rate" of $p$; the probability of committing the particular crime undetected. Recognizing (to the best of our knowledge) the unanimous finding in the literature, we assume that $p>\frac{1}{2}$; namely that financial misconduct is more likely to go undetected. Furthermore, given the sparse and ambivalent results in the literature on whether financial frauds pay off on average (which we outline in greater detail below), we adopt the simplifying assumption that they yield an expected payoff of $0^{2}$. Note that this simple representation of crime already characterizes a menu of criminal activities to choose from. Some crimes $(p \approx 1)$ are "petty", like a modest earning manipulation that barely transgresses the line between legal and illegal. These will most likely yield only modest payoffs even when successful, yet is also very likely to go unpunished, and are represented by high values of $p$. Other forms of crime are more "serious", like a large-scale misreporting which will prove lucrative if successful, but also proportionately more likely to get caught $\left(p \approx \frac{1}{2}\right)$, especially if the scheme is large in scale. These are represented by lower values of $p$.

With this "choice set" of crime and the pressure-based dis-continuity we introduced, we first examine a "cliffhanging" situation. In the context of the Coca-Cola example, suppose the manager believes that the company can narrowly meet the analyst forecast, but any negative shock in demand would precipitate a fall below. It is then easy to intuit the urge to engage in a gamble which will, in the vast majority of cases, allow the firm to stay above the cliff (e.g., withstand the negative shock in demand), at the cost of a large, but less frequent fall when caught. This gamble is a negatively skewed bet, much like the act of selling a "deep out-of-the-money" put option. The manager's intense focus on exceeding the target (the "tunnel vision") generates a preference for a gamble that delivers a frequent but modestly positive payoff so as to stay above the target, at

\footnotetext{
${ }^{2}$ This assumption could easily be relaxed given the parsimony of our model, but serves as a good starting point from which the results would not vary materially by adding modest positive or negative expected payoffs.
} 
the expense of a less frequent but commensurately large fall, the depth of which is of second order concern compared to the far more imperative goal of staying above the target. We show that this urge is an unequivocal incentive to misbehave, arising as an optimal response to the "tunnel vision" to attain the target. And as is perhaps also intuitive, the degree to which agents seek negative skewness depends on how precarious the situation is, a statement we make more precise. It is important to note that the incentive to misbehave in our model is not the consequence of a positive expected payoff associated to crime; in fact, it would hold for (modestly) negative expected payoffs as well. We have simplified the first moment to be null in our "choice set" of crime following the weak evidence on either side, and focus instead on the third moment - skew-ness - whereby the evidence $\left(p>\frac{1}{2}\right)$ seems unanimous in the literature. Our focus on the third moment, whilst novel on its own, also proves to be a natural complement to the dis-continuity that represents a sense of desperation, the signs of which is ubiquitously scattered about in the literature as well as in practitioners' accounts.

Our model is parsimonious, yet powerful enough to generate several interesting empirical predictions. We show a "pecking order" in the preference for crime. Namely, the "petty" crime will be depleted first, and only when these quasi-legal measures are depleted, will the agent turn to more "serious" violations. We also show that the "petty" crime is likely be pervasive, albeit less detectable, leading to a somewhat uncomfortable conclusion that infringement of the law is the norm, rather than the exception, in the business world. Our model also predicts that the most serious violations will be concentrated around the "cliff" rather than in aspirational situations where the target is an ambitious goal to attain. This means that the empirical evidence for pressure-based stories would likely be stronger than narratives related to "capital market incentives" such as the attainment of executive compensation targets. Our model is also conducive to explaining the notion of "slippery slope" to financial misconduct. As the distance from the cliff decreases from above, the agent is gradually channelled into an increasing incentive to misbehave. While many of these predictions agree with existing empirical research on the subject, many still lack matching empirical tests, leaving room for further empirical investigation. Also, our model develops a "framework" that very much reminisces those used in standard micro-economics, a reason we believe our model is also amenable to further work on the theoretical side. Our framework highlights currently overlooked, yet intuitively important elements, such as the "scale" of crime and a "menu" of crime to chosen from, providing a novel theoretical perspective on financial fraud. 
In economics and related disciplines, analyses on criminal incentives often follow the spirit of Becker (1968), with a utility maximizing agent calculating the costs and benefits related to the commission of crime. While this framework is natural, there are at least two reasons why the use of Becker's framework is not altogether satisfactory in the context of financial misconduct. First, the Becker framework inevitably requires a rather strong assumption that the net expected payoff (benefit minus cost) from crime must exceed the required compensation for risk. In the case of financial misconduct however, the literature finds little evidence of such positive (unconditional) expected net payoff: if anything, the findings are ambivalent and incomplete at best, ${ }^{3}$ thus it seems unlikely that perpetrators commit to crime under the calculated conviction that it would pay off on average $^{4}$, as they should in the Becker framework. Our skepticism is deepened by Soltes (2016) who finds that those involved in white-collar crime typically do not seem to weigh the expected costs against expected benefits ${ }^{5}$.

Secondly, the conventional usage of Becker's framework does not accommodate the possibility of non-monetary utility gains from exceeding a target. Of course, the utility jump we consider may as well be ascribable to monetary gains, for example, the attainment of a 'milestone' that delivers a large monetary compensation. However, non-monetary reasons are also entirely conceivable, as is the case in the example from Soltes (2016) where the forecast became a target simply because "it was an endorsement that we were doing the right things". What is more, given the widespread evidence on value-destroying short-terminism related to the attainment of salient targets (e.g., Bhojraj et al., 2006, or Graham, Harvey and Rajgopal 2005) -despite all the strenuous efforts to align compensation schemes with the value of the firm in the longer term- it seems only prudent to incorporate the possibility that there may be factors other than just pecuniary gains at play,

\footnotetext{
${ }^{3}$ Some find that the financial misconduct incur significant penalties in the form of job loss (Karpoff, Lee and Martin 2008) and/or poor subsequent job prospects (e.g., Arthaud-Day et al. 2006, Desai, Hogan and Wilkins 2006, Hennes, Leonne, and Miller 2008). On the other hand, a more recent study by Amiram, Huang and Rajgopal (2020) find that $32.9 \%$ of perpetrators experience positive payoff from commission of misconduct even when they are caught, but falls short of an full-fledged unconditional assessment.

${ }^{4}$ Otherwise, one needs to assume that all perpetrators are inexorably risk-seeking. The financial misconduct literature seems to have found this to be either implausibly strong, or the risk aversion of criminals to be of secondary importance (or too difficult to estimate), and has established a tradition of looking almost exclusively at the first moment (expected payoffs) rather than discuss in depth the consequences of risk aversion of criminals.

${ }^{5} \mathrm{He}$ writes, after interviewing 48 former executives spanning seven years: "Many people, federal prosecutors, scholars, and media commentators claim that executives make decisions, including criminal ones, through explicit cost-benefit calculation. Although such deliberate reasoning is consistent with the way many business decisions are made, this explanation seems at odds with how these former leaders made choices that eventually led them to prison. Many were not mindfully weighing the expected benefits against the expected costs"
} 
quite possibly in the form of managerial pressure. These non-monetary sources of satisfaction can only be represented as utility jumps, rather than changes along the utility function, yet Becker's framework typically suppresses the possibility of jumps, by almost always restricting itself to using either a completely linear (risk-neutrality) or nearly linear (e.g., power utility) forms of utilities with arguments that only involve monetary terms. Our model is agnostic about the origin of the pressure, hence accommodates a wider range of managerial pressure in the form of both monetary and non-monetary sources, with a unifying focus on the dis-continuity they engender. Our focus on dis-continuity -together with the notion of skewness-seeking behavior- provides a new and potentially powerful perspective in the analysis of financial misconduct.

\section{Setup}

In this section, we set up the model describing the optimal choice of misconduct, in the context of a manager who is facing managerial pressure. There are two main components: $(i)$ a notional utility function of the manager (denoted as $v_{T}(X)$, and called "valuation function") with a focus on a dis-continuous drop in utility if the managerial goal is not met, and (ii) the set of crime that the manager can choose from (denoted as $M_{b}(p)$, called "binomial martingales").

\section{$2.1 \quad X$ : Manager's Variable of Interest}

Consider again the example of SEC's investigation of Coca-Cola in 2005, where the manager's interest was to ensure that the revenue of the firm exceeds analyst forecasts. Clearly, revenue in this example was a "variable of interest" to the manager. We denote such variables of interest generically as $X$. Presumably, $X$ is relevant to the firm's value and hence is of public/investor's interest as well, but in the context of fraud, $X$ is also likely to be amenable to some degree of manipulation by the manager, for instance, in the form of "accounting hocus-pocus" or voluntary disclosure. Other such examples of $X$ could include: the firm's stock price, earnings per share, performance indicators such as revenue or market share, the amount of capital raised, the credit rating given to the firm, the firm's known progress on its core project, and the like.

Given today's observed $X$, (denoted as ' $x$ ') the manager is concerned about the value of $X$ tomorrow $^{6}$ ( $X^{\prime}$; e.g., the revenue of Coca-Cola next quarter), in particular, the likelihood of $X^{\prime}$ falling

\footnotetext{
${ }^{6}$ Regarding the notation of $X$, we follow the tradition of capitalizing the random variable itself, and denote its realized value by lower-case letters.
} 
below an imperative managerial goal ( $T$; e.g., Wall Street's revenue forecast for Coca-Cola next quarter), in the event of a negative shock. That is, the manager is worried about the possibility of $X^{\prime}<T$ arising, where

$$
X^{\prime}=x+u, \quad \mathbb{E}(u)=0,
$$

a simplest expression of the fact that there is some uncertainty involved in predicting the value of $X^{\prime}$ tomorrow given today's $x$. The dreaded event of $X^{\prime}<T$ is more likely the more negative the realization of uncertainty $(u)$, presumably generating a stronger incentive to misbehave, but we assume for conservatism and simplicity that the uncertainty is neutral on average. $(\mathbb{E}(u)=0)$. Other than this, we intentionally assume very little more ${ }^{7}$ on $u$. This is because a typical criminal situation would most likely involve 'Knightian uncertainty', in the sense that the distributions of the associated random components are neither precisely known nor published, hence we would like our results to be robust to a vast array of conceivable distributions. In short, it is neither the technical properties, nor the precise shape of the distribution of $u$ that is driving our results.

\section{$2.2 v_{T}(X)$ : Valuation Function with a "Cliff"}

$X$ is a "variable of interest" to the manager, and this interest -with a particular focus on whether the target $T$ is attained or not- is quantitatively represented by $v_{T}(X)$ :

$$
v_{T}(X):=(-1) \cdot \mathbb{1}_{\{X-T<0\}}+0 \cdot \mathbb{1}_{\{X-T \geq 0\}},
$$

pictorially described as the black graph on Figure 1.

If the manager is facing a situation where $x \geq T$, and is concerned about the possibility of $X^{\prime}<T$ arising tomorrow, (s)he would be sitting on a 'buffer' of amount $B:=x-T \geq 0$ to spare against a potential negative shock of $u<0$ pushing her down the the dis-continuous drop at $X=T$. This would be effectively describing a "cliffhanging" situation. Conversely, if $B<0$, (s)he would be 'behind' the precipice, describing an aspirational situation where the goal remains yet to be attained. Naturally, each valuation function $\left(v_{T}\right)$ is indexed by $T$, the location of the target.

Clearly, this step-like representation of $v_{T}$ is a simplification of reality with an intendedly explicit

\footnotetext{
${ }^{7}$ The additional assumptions we adopt are technical in nature, so as to enable 'analytic' comparative statics results. Specifically, the $p d f$ of $u$ is single-peaked, continuously differentiable, symmetric, finite-varianced, and satisfies the 'single-crossing property' outlined in the Appendix. Throughout, the assumptions remain general enough so as to encompass the vast majority of densities that are used in economics.
} 


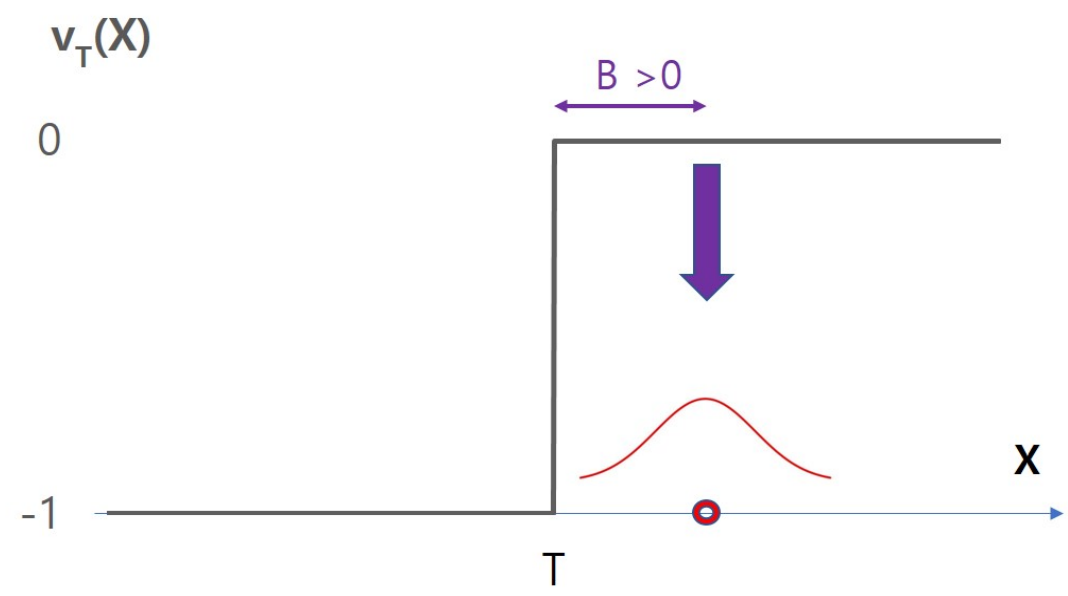

Figure 1: $v(\cdot)$ with some buffer to spare $(B>0)$

focus; the pressure from missing the managerial target $\left(X^{\prime}<T\right)$. This focus is corroborated by the myriad findings in the literature and practitioner accounts that either directly or indirectly testify to the presence of managerial pressure. Some sources of pressure are 'real'. For example, $T$ could matter because "people (CEO's) are paid not for what they do but for what they do relative to some target" (Jensen, 2005) and also because "the capital market punishes the entire firm if they miss analysts' forecast by as little as a penny" (Jensen, 2005). Similarly, T may be 'real' because it represent the amount of temporary bridge-funding needed to avoid bankruptcy, or the amount that needs to be financed to initiate a large-scale and bulky investment. Meanwhile, other accounts appear to be more psychological in nature, for example, the statement by a former SEC Chairman (Levitt, 2002): "what is driving them is the desire to see to it that, quarter by quarter, their earnings reach levels of a penny or two or three more than the quarter before that", alluding to a form of managerial hubris (Malmendier and Tate, 2005) that (s)he "can and will make progress no matter what". In similar psychological vein, $T$ may represent a target that needs to be met to deal with internal governance pressure (Feng et al, 2011), to consolidate the CEO's standing in the firm's internal politics, or perhaps to earn respect from subordinates. And also quite possibly, the focus on $T$ may derive from a combination of real and psychological reasons, such as beating the performance of a competitor (e.g., duopoly). While the nature of these pressures may vary from the most real/monetary to psychological -and anything in between- they all share the commonality of a "tunnel vision" (Chu et al. 2019) around $T$ and a presumable drop of utility if left 
unaccomplished, which we capture by the "cliff" at $X=T$.

Lastly, we discuss possible modifications of $v_{T}$. While the sharp discontinuity of $v_{T}$ is probably a reasonable abstraction of reality in the majority of cases (e.g., compensation milestones or analyst forecasts), there could be situations where gradual ("mollified") increments may seem more adequate (e.g., pressure from multiple peers, or multiple investments), as is illustrated on the left panel of Figure 2. Similarly, one may also argue it is unrealistic to assume that increments occur solely in discontinuous jumps, and therefore warrants a combination of continuous and discontinuous representation as illustrated on the right panel of Figure 2. We concentrate on, and draw analytic results based on the original representation, but we suspect that much of our results carry over to the suggested alternative representations as well. ${ }^{8}$ This is so, because in the original and modified versions alike, the key aspect is that they are piece-wise convex and concave, which is the driving force of the results we find.
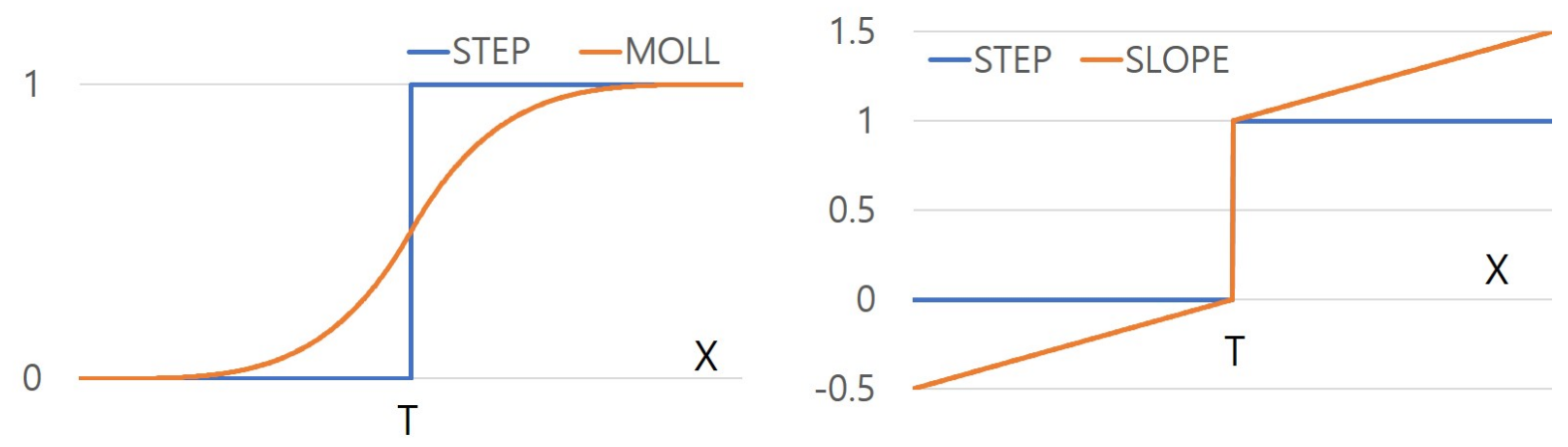

Figure 2: $v_{T}$ : original and modified versions

\section{$2.3\left\{M_{b}(p): p>\frac{1}{2}\right\}:$ The "Choice Set" of Financial Misconduct}

Consider a manager, who observes current revenue $(x)$, contemplating the illegal act of "channel stuffing". Quite naturally, the consequence of this act would depend critically on whether the scheme becomes detected or not. We denote the "success rate" of crime -the likelihood of committing the crime undetected- by $p$. If undetected, the act will provide a deceptive boost in perceived revenue $\left(X_{\text {success }}\right)$, whereas detection would result in a state $\left(X_{\text {failure }}\right)$ worse than the truthful

\footnotetext{
${ }^{8}$ Albeit quite possibly with loss of analyticity, which is why we chose not to pursue these representations in this paper.
} 
counterfactual $(x)$ due to disciplinary actions from the authority, as well as the stigma it entails. Therefore, $X_{\text {failure }}<x<X_{\text {success. }}$. We report two sets of findings in the literature that allow us to be more specific about $X_{\text {success }}$ and $X_{\text {failure }}$.

First, the literature finds little evidence that the expected payoff (benefit minus cost) of financial crime is positive. The only directly relevant study we know of in this regard is by Amiram et al. (2020), who estimate a $52 \%$ chance that the expected return is positive (and $48 \%$ chance it is negative), albeit with a large " $10 \%$ confidence interval" of $[34 \%, 76 \%]^{9}$. Other studies are less direct, some results emphasizing potential costs of detected misconduct such as poor subsequent job prospects (e.g., Arthur-Day et al. 2006, Desai, Hogan and Wilkins 2006, Hennes, Leonnes and Miller 2008) or job loss (Karpoff, Lee and Martin 2008) and others highlighting the potential benefit (e.g., Dichev et al. 2003, Eisinger 2017, Amiram et al. 2020), but as a whole, seem evenhandedly ambivalent. Therefore, given the current state of research on this matter, we adopt the simplifying assumption that $(i)$ the expected payoff of crime is zero ${ }^{10}$. Secondly, the literature seems unanimous $^{11}$ in reporting that the likelihood of detection of financial misconducts is small, typically well below $50 \%$. In the context of our model, this means a $(i i)$ high "success rate" of crime: $p>\frac{1}{2}$, another assumption we adopt.

Imposing the first assumption (net zero expected payoff) implies that a unit gamble of crime yields ${ }^{12}$ payoffs as illustrated in Figure 3. We denote these gambles - with probability of success $p$ and zero expected payoffs- by $M_{b}(p)$ and call them binomial martingales, since their payoffs are binary and represent fair gambles (i.e., martingales). Suppose the manager enters into $N$ units of such gambles while observing $x$ (e.g., current revenue), where $N$ represents the scale of crime (e.g., the amount of "channel stuffing" to be done). Since entering a gamble of scale ' $N$ ' would yield the payoff of

\footnotetext{
${ }^{9}$ This is with some abuse of terminology, as this band is given by varying the probability of detection rather than by randomness in the statistical sense of the word. We refer the reader to Amiram et al. (2020) for a precise description of their results.

${ }^{10} \mathrm{As}$ a starting point, we believe this is a good representation of the current state of research on this issue. Meanwhile, our model can certainly be extended to incorporate positive or negative first moment if the literature arrives at a different consensus, but we strongly suspect that the results would not be altered significantly.

${ }^{11}$ We are not aware of any findings in the literature that claim $p<50 \%$ in the context of white collar crime. Dyck et al. (2020) provide a most recent and direct estimate of detection rate at $31 \%$ which amounts to $p=69 \%$ in the context of our model. Zakolyukina (2018) estimates $p \approx 97 \%$ per year. A case study (Shapira and Zingales, 2017) on corporate misbehavior by DuPont estimates a figure around $80 \%$.

${ }^{12}$ The unit of gamble has only two possible outcomes, denoted by $\pi_{\text {success }}$ and $\pi_{\text {failure }}$, with probabilities $p$ and $1-p$ respectively. Without loss of generality, we normalize the size of the bet: $\pi_{\text {success }}-\pi_{\text {failure }}=1$. By zero expected payoffs, we need $\pi_{\text {success }} \times p+\pi_{\text {failure }} \times(1-p)=0$. Solving the system of equations pins down $\pi_{\text {success }}$ and $\pi_{\text {failure }}$.
} 


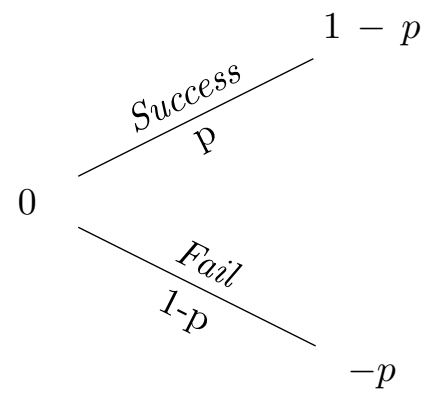

Figure 3: Payoff on a Unit Gamble of Crime (Binomial Martingale)

' $N \times M_{b}(p)$ ' that adds upon the "truthful counterfactual" $x$ (i.e., the gamble would generate the payoff of ' $x+N \cdot M_{b}(p)$ '), it is easy to see that:

$$
x-N p=X_{\text {failure }}<x<X_{\text {success }}=x+N(1-p),
$$

where $X_{\text {failure }}$ arises with probability $1-p$ and $X_{\text {success }}$ arises with probability $p$. Furthermore, imposing the second assumption $\left(p>\frac{1}{2}\right)$ informs us that crime is typically chosen to be a "small yield but safe option" in the sense that the potential gain $N(1-p)$ is outweighed by the potential loss $-N p$, but this loss occurs commensurately less frequently. Taken together, the set of binomial martingales, $\left\{M_{b}(p): p>\frac{1}{2}\right\}$, denotes the typical "choice set" of financial misconduct that a manager ends up choosing from.

\subsection{The Choice of Skew-ness: $p^{*}$}

In order to contextualize the choice of $p^{*}>\frac{1}{2}$, consider the payoff structure of $M_{b}(p)$ in Figure 3 . When $p=0.5, M_{b}(p)$ becomes a familiar symmetric bet where the upside $(+0.5=1-p)$ and downside $(-0.5=-p)$ are equally likely, much like regular stocks. When $p=0.01$, the upside $(+0.99)$ is large and the downside $(-0.01)$ is limited but the chance of an upside is low (with probability 0.01). This is a positively skewed gamble, like the purchase of a lottery ticket. When, $p=0.75$, a modest gain $(+0.25)$ is more likely (with probability 0.75$)$ than a large but infrequent loss $(-0.75$, with probability 0.25 ), and this represents a negatively skewed gamble. These examples illustrate a general fact (Aristidou et al., 2021): the success rate $(p)$ of binomial martingales uniquely controls the level of skew-ness of its payoff structure, where $p<\frac{1}{2}$ represent positively skewed bets, and $p>\frac{1}{2}$ represent negatively skewed bets. We express this relationship graphically in Figure 4 where darker color represents higher probability of outcomes in various economic choices. 


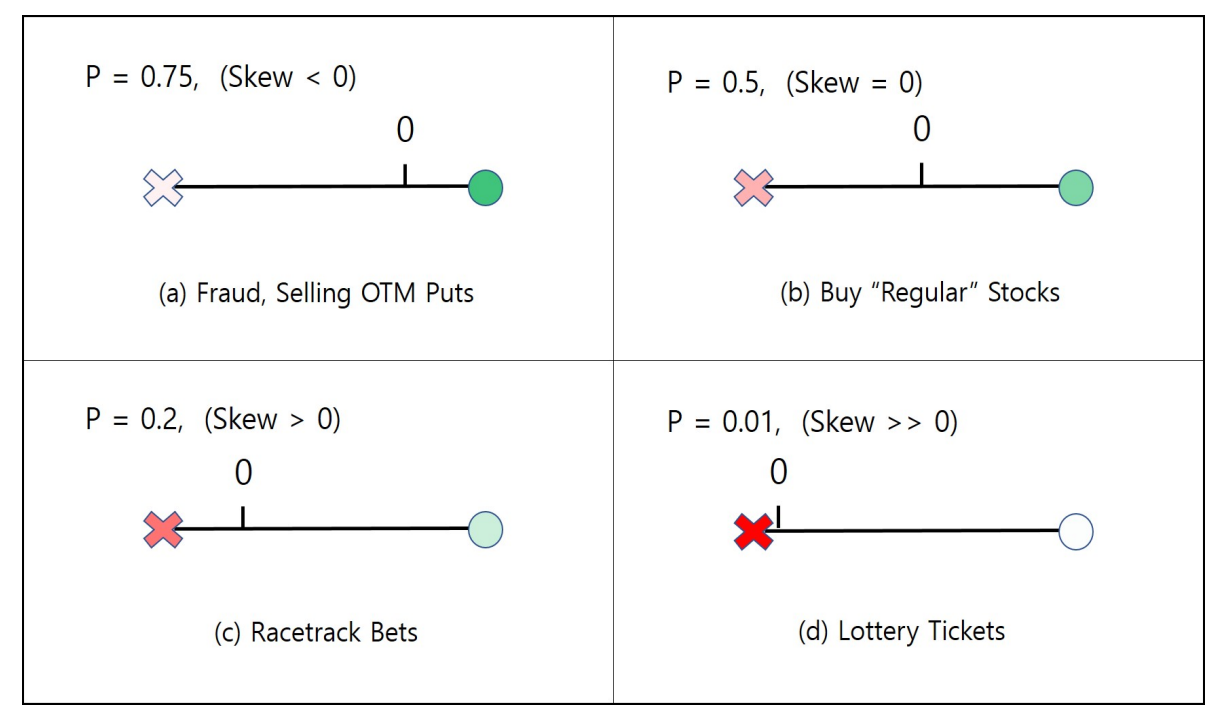

Figure 4: Skewness Embedded in Various Economic Choices

The aforementioned equivalence of

$$
p>\frac{1}{2} \Longleftrightarrow \text { Negatively skewed bets, }
$$

along with the unambiguous choice of $p^{*}>\frac{1}{2}$ reported in the literature, begs the question: Why does financial misconduct manifest itself in the form of negatively skewed gambles, as opposed to positively skewed ones? We will provide a clear answer by allowing an optimizing agent to respond to managerial pressure $\left(v_{T}\right)$ and observing the optimal choices. It turns out that our focus on skew-ness -which in fact followed naturally from incorporating the findings in the literature in the form of binomial martingales- is neither coincidental nor contrived; it is fundamental to analyzing the behavior around pressure points $(T)$.

\subsection{The Optimization Problem}

The problem is standard optimization under uncertainty, much like portfolio allocation, with the aim of maximizing $v_{T}\left(X^{\prime}\right)$, the value of $v_{T}$ tomorrow. The manager observes $x$ today, and chooses $p$ ("success rate" of crime) and $N$ ("scale" of crime). As per the payoff structure described in (2), the manager modifies (1), and rationally expects $X^{\prime}$ (the value of $X$ tomorrow) to be:

$$
X^{\prime}(N, p)=x+N \cdot M_{b}(p)+u, \quad \mathbb{E}[u]=0 .
$$


In the narrative of the Coca-Cola example, the manager observes the revenue today $(x)$ and desires to avoid $X^{\prime}<T$. If the firm is managed "truthfully" (i.e., $N=0$ ), the revenue next quarter would be only an uncertainty away from today's $\left(X^{\prime}=x+u, \mathbb{E}[u]=0\right)$. But if the manager is concerned that the downside of this uncertainty may push the revenue below the Wall Street forecast $\left(X^{\prime}<T\right)$, (s)he may be tempted to adopt a martingale gamble of scale $N>0$ (so that $X^{\prime}=x+N \cdot M_{b}(p)+u, \mathbb{E}[u]=0$ ) which, if all goes to plan (with probability $p$ ), would give an apparent boost in revenue so as to alleviate the downside risk.

The optimization problem ${ }^{13}$ is then to maximize $\mathbb{E}\left[v_{T}\left(X^{\prime}(N, p)\right)\right]$ over $p$ and $N$, namely:

$$
\max _{\substack{p \in(0,1) \\ N \in[0, \infty)}} \mathbb{E}\left[v_{T}\left(X^{\prime}(N, p)\right)\right]
$$

where

$$
X^{\prime}(N, p)=\left\{\begin{array}{l}
x+N(1-p)+u, \quad \text { with probability } p \\
x-N p+u, \quad \text { with probability } 1-p
\end{array}\right.
$$

Note that there is no 'budget constraint' on this optimization because $M_{b}(p)$ is costless to set up. There is no restriction on $p$ either (i.e., not restricted to $p>\frac{1}{2}$ ), as the observed preference for negative skew-ness $\left(p^{*}>\frac{1}{2}\right)$ will arise as a consequence of this optimization problem. In the context of the "cliff" at $X=T$ (Figure 1), the optimization problem is to cleverly choose from an unrestricted menu of martingale gambles that can come in all "skew-ness (as shown in Figure 4) and sizes", so as to either (1) maximize her chances to remain on the cliff if one starts with the "cliffhanging" position $(B>0$, or equivalently, $x>T)$ or to $(2)$ maximize her prospects of jumping above the cliff if one were to start from an "aspirational" situation $(B<0$, or equivalently, $x<T)$. The question then is to explore and interpret these choices under various parameter configurations, which is the goal of our next section.

\footnotetext{
${ }^{13}$ Strictly speaking, mathematically, (4) is 'ill-posed', simply because the domain is not a compact set, which is trivial to mend. But the very fact that (4) is ill-posed -as well as the proposed remedy- has economic content, so for the sake of exposition, we will start with this setup and modify it as we explain the economic significance of its modification. The strategy is to discuss general properties of economic choice regarding the maximization of $v_{T}$ irrespective of the domain, and then to consider restrictions on the domain.
} 


\section{The Incentive to Misbehave}

We start the analysis from the "cliffhanging $(B \geq 0)$ " case. In Proposition 1, we show the connection between preference for skew-ness and the dis-continuity point at $T$, a useful intuition that permeates throughout the paper. We then provide a result (Proposition 2) that uncovers an inevitable trade-off that the manager is bound to face while making the optimal choice of financial misconduct.

\subsection{The Desirability of Negative Skewness: "Pushing the Peak Forward"}

We first show that any agent seeking to maximize $\mathbb{E}\left[v_{T}\left(X^{\prime}(N, p)\right)\right]$, which we call " $E V$-maximizer", would unambiguously prefer negatively skewed binomial martingales. Proposition 1 provides a first glimpse of fraudulent incentives arising as an act of (cliffhanging) desperation, an explanation for the pervasive findings of $p^{*}>\frac{1}{2}$ in the literature, and a justification for our characterization of $\left\{M_{B}(p), p>\frac{1}{2}\right\}$ as the typical "choice set" of crime.

Proposition 1 (Preference For Negative Skewness). Let $B \geq 0$. Suppose an EV-maximizer was observed choosing $(\hat{N}, \hat{p})$. Then $\hat{p} \notin\left(0, \frac{1}{2}\right]$

Proof. See appendix.

Intuitively, the preference for negative skewness can be easily understood with a particular case: normally distributed uncertainty $\left(u \sim N\left(0, \sigma^{2}\right)\right)$. Consider as an example, the simple situation where the agent is perched right on top of the cliff $(B=0)$, facing a normal uncertainty $u$ which may push her below the cliff upon a negative realization of any size $(u<0)$. Now suppose the agent has the freedom to 'deform' the probability distribution (s)he faces, but under the restriction that the mean $(\mathbb{E}(u)=0)$ must be preserved. It is then easy to visualize that the likely choice will be to push forward the peak (originally at 0, given normality) slightly forward so as to assign more probability mass to the event of $X^{\prime}$ narrowly exceeding $T$, as illustrated in Figure 5 below. And given the restriction that the mean must be preserved, this deformation could only be achieved at the expense of a fatter lower tail ${ }^{14}$ which, in the language of statistics, spells a negatively-skewed distribution ${ }^{15}$.

\footnotetext{
${ }^{14}$ Note that under our discontinuous but otherwise flat $v$, agents only care about whether they are below or above $T$. This makes them indifferent to the presence of fatter or thinner tails, and this indifference allows agents to optimize using skew-ness. Of course, the results we present may quantitatively change by relaxing this indifference assumption -for example by using a $v$ depicted in the right panel of Figure 2- but we still think much of the results will still carry over qualitatively for the aforementioned reasons.

${ }^{15}$ In our simplistic model, we do not deform the distribution of $u$ as in Figure 5, which in fact is what is known as skewed normal distribution whose analytical properties are not transparent. In our model, there are two sources of uncertainty: $u$, which is by definition symmetric, and $M_{b}(p)$. The deformation is given by a 'mixing' the two, or more formally as a compound lottery of these two gambles. While this is not the same as an outright deformation
} 


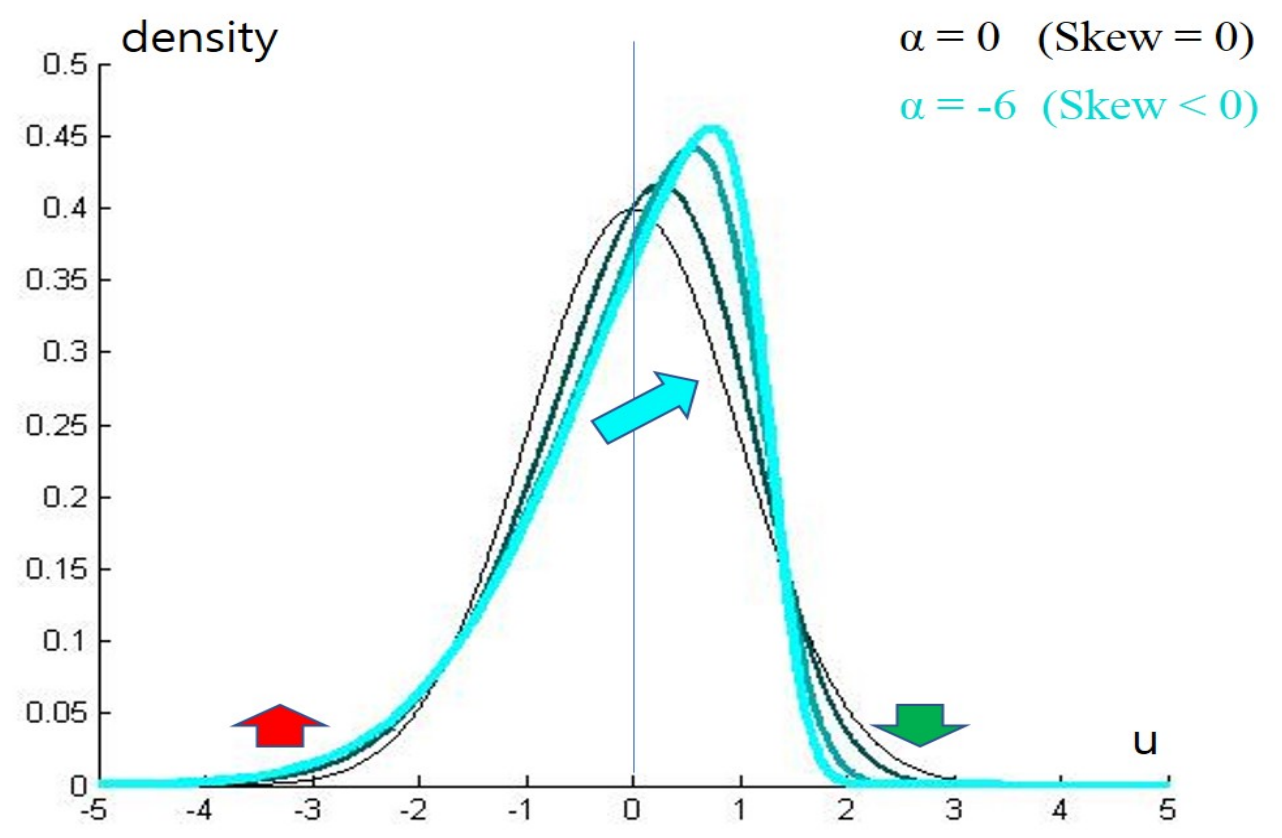

Figure 5: The Motive to Skew the Distribution: Unequivocally Negative

Despite the visualization of "pushing the peak forward", we emphasize that this result is not about positive expected payoffs associated to fraud; after all, it must come at the expense of a "fatter tail". We have intentionally suppressed all our random components, $u$ and $M_{b}(p)$, to be fair gambles for the sake of transparency, and have shown that the incentive for misbehavior can arise even without postulating a positive expected payoff. Thus, a key value of Proposition 1 is that it urges us to shift the some of the focus given to the expected payoff -i.e., the first moment- of fraud to what we believe could be another plausible and significant motivator of fraud schemes, -i.e., the third moment- by showing that agents endogenously develop unequivocal preference for negative skewness under a cliffhanging situation. However, our restrictive attention on martingales is not to be construed as a refutation of Becker's framework (1968) -which the literature seem to have commonly interpreted as suggesting cost-benefit analyses on the first moment- as this restriction is adopted for the sake of focus. In fact, we envision a straightforward generalization into submartingales or super-martingales, which would then be be a downright enrichment, rather than a refutation, of Becker (1968).

of $u$, it still carries the same key message of "skewing the uncertainty to address discontinuous payoffs" and retains transparency to enable analytic results. 
The overarching sentiment that underlies the action of "pushing the peak forward (at the expense of a fatter lower tail)" can be interpreted as one of desperation. The notion of desperation is, in fact, a recurrent theme scattered about in the fraud literature. As an example, representing numerous others in the same spirit, Chu et al (2019) write: "similar to athletes engaging in competition, they experience "tunnel vision" - that is, they lose sight of the big picture and do "whatever it takes" to meet their goal... Ultimately, in some firms, executives become so desensitized to their accounting choices that they move "outside of GAAP" to beat earnings expectations." Similar accounts are prevalent among practitioners as well, for example, as in the statement from the earlier quote in Soltes (2016): "my goal was just to get to or over that number-and if I did that, I succeeded." The prevalence of such notion notwithstanding, the theoretical literature has yet to provide a framework to understand its plausible but unexplored connection with fraudulent acts. Our attempt is novel in being the first to capture this sentiment in a model, and in showing how the agent's intense and desperate focus on $T$ concentrates the agent's actions upon staying above $T$ while ignoring the consequence of failure. Proposition 1 unambiguously shows that these considerations propel the agent towards an attempt to "push the peak forward," an endeavor that manifests itself as preference for negative skew-ness, namely, an incentive to misbehave.

\subsection{The Trade-off Between "Gain" and "Precision"}

Given this preference for negative skew-ness, we proceed by asking how much negative skew-ness agents would choose. Proposition 2 below answers this question: they opt to be as negatively skewed as possible, but under a condition. The underlying mechanism behind these choices consist of a combination of two elements: (1) an inevitable "trade-off" that the manager must face in the commission of crime, and (2) the "scale" of crime $(N)$. We explain each sequentially after introducing Proposition 2.

Proposition 2 (Most Negatively Skewed Bet). Let $B \geq 0$.

(i) Consider a monotone sequence $\left\{p_{k}: \frac{1}{2}<p_{k}<1\right\}_{0}^{\infty} \uparrow 1$. For any such sequence, there exists a corresponding sequence $\left\{N_{k}\right\}_{0}^{\infty}$ such that $\left\{\mathbb{E}\left[v_{T}\left(X^{\prime}\left(N_{k}, p_{k}\right)\right)\right]\right\}_{0}^{\infty}$ is strictly increasing in $k$.

(ii) Moreover, we can always find $\left\{\left(N_{k}, p_{k}\right)\right\}_{0}^{\infty}$ such that $\mathbb{E}\left[v_{T}\left(X^{\prime}\left(N_{k}, p_{k}\right)\right)\right] \stackrel{k \rightarrow \infty}{\longrightarrow} 0$.

Proof. See appendix.

Proposition 2-( $i$ ) tells us that the agent can always do better by combining two strategies: buy- 
ing a more negatively skewed bet accompanied by scaling up the negatively skewed endeavor. In the language of a fraud scheme, the agent will prefer a fraud scheme whose payoff is modest but more likely to go unnoticed if such a scheme can be committed at a large enough scale. Furthermore, Proposition 2-(ii) tells us what such a strategy could ultimately achieve. Recalling that $\mathbb{E}\left[v_{T}\left(X^{\prime}\right)\right]=0$ would be the expected value of $v_{T}$ if the the cliff did not exist at all, Proposition 2-(ii) simply states that the agents can shield herself completely from the precipitous drop as long as the combination of strategies are available at an arbitrarily large scale. In sum, Proposition 2 states that $p$ and $N$ are complements, hence, as long as the agent has access to a full range of both dimensions, (s)he will demand the most negatively skewed bet, asymptotically negating the presence of the cliff altogether as $N$ becomes arbitrarily large.

A first step towards understanding the choice depicted in Proposition 2 is to focus on a tradeoff that arises from a rather natural preference; that an "ideal act of crime" should not only be gainful, but also undetectable. That is, the potential perpetrator values both (1) the "gain" from the fraud - the size of payoff from a successful execution of crime - as well as (2) the "precision" in executing the fraud - the likelihood that it will go undetected. But recapitulating the result in Figure 3 showing that the "gain" to a unit ${ }^{16}$ of crime, $M_{b}(p)$, is ' $1-p$ ' whereas its "precision" is ' $p$ ', these two desirable aspects are clearly in conflict. Quite understandably, a criminal option that is both gainful and undetectable is "too good to be true" or, in our model parlance, not martingale. Therefore, the optimal choice of $p$ must invariably represent a compromise within this trade-off between "precision $(p)$ " and "gain $(1-p)$ ". In the context of understanding Proposition 2, the question then is: how does altering the "scale" of crime $(N)$ affect this trade-off?

\subsection{The "Scale" of Crime $(N)$}

Note that this trade-off is more dire when $N$ is small. Recalling that the "gain" from deploying $N$ units of $M_{b}(p)$ is $N(1-p)$, it is easy to see that the choice of $p$ that is required to secure an identical magnitude of gain, $\overline{N(1-p)}$-the size of which is important since this is, after all, what protects the manager from falling off the "cliff"- would differ by the scale of crime, $N$. For instance, the choice of $p$ to secure a unit of gain $(\overline{N(1-p)}=1)$ is $p=0.9$ when $N=10$, as opposed to $p=0.8$ when $N=5$. Evidently, the agent is forced to relinquish more of "precision" (i.e., choose a lower $p)$ to secure a given amount of gain $(\overline{N(1-p)}=1)$ when $N$ is low (i.e., $p=0.8$ when $N=5$ vs

\footnotetext{
${ }^{16}$ In this paragraph, we set $N=1$ for the sake of focus.
} 
$p=0.9$ when $N=10$ ), making the trade-off between "gain" and "precision" more binding as $N$ becomes lower. And symmetrically, one can alleviate this trade-off by scaling up the fraud scheme. In fact, in the current setup ${ }^{17}$, one can enhance the "precision" of fraud without sacrificing the magnitude of "gain" at all, by increasing $p$ to an arbitrarily high level ${ }^{18}$ as is desired $\left(p^{\prime}>p\right)$, while scaling up the scheme $(N)$ to the correspondingly necessary magnitude $\left(N^{\prime}=\frac{\overline{N(1-p)}}{1-p^{\prime}}\right)$ so as to preserve the size of "gain". ${ }^{19}$ The efficacy of this joint maneuver of scaling up $p$ and $N$ together is the underlying mechanism that drives the results in Proposition 2-(i) and (ii). And this also effectively makes $p$ and $N$ complements, an intuition we make explicit in Proposition 4.

Of course, the application of this joint maneuver comes at the price of a more disastrous outcome for $X^{\prime}$ in the case of detection. Namely, the payoff upon a failed commission of crime $\left(X^{\prime}=x-N p\right)$ would be lower if $N$ is replaced by $N^{\prime}>N$. However, this lowered payoff does not incur any utility cost as $v_{T}$ is flat everywhere below $T .{ }^{20}$ Thus, the act of scaling up the fraud scheme $(N)$ can effectively be employed as a "high-powered lever" to override the trade-off, precisely because the price they pay -the lowered payoff in the case of failure- is of relatively secondary order concern compared to the more imminent task of "clinging onto the cliff". In this sense, it is this urgency of attaining $T$ - and consequently, the relative insensitivity ${ }^{21}$ to the depth of the fall (i.e., the magnitude of $\left.\left|X^{\prime}-T\right|\right)$ - that enables $N$ to act as an effective lever, the process which underlies Proposition 2. And clearly, this leverage action must be executed in conjunction with the skew-ness seeking behavior we outlined in Proposition 1, a reason why we believe the analysis of skew-ness is essential in the context of financial crime. (See Example 3.4 below.)

Our analysis of the trade-off, along with the fact that $N$ is a key determinant of the stringency of

\footnotetext{
${ }^{17}$ Recall that in the current setup, agents are free to choose $p$ and $N$ anywhere within its range: $p \in(0,1)$ and $N \in[0, \infty)$.

${ }^{18}$ For those readers who are more visually oriented, this can be visualized in Figure 4 by slowly dragging the barbell to the left (as one could by progressing from panel ' $d$ ' to panel ' $a$ ') to make the green light shine brighter.

${ }^{19}$ That is, choose the value of $N^{\prime}$ such that the relationship $N^{\prime}\left(1-p^{\prime}\right)=\overline{N(1-p)}$ is honored so as to fix the magnitude of "gain" while ensuring higher "precision" $\left(p^{\prime}>p\right)$.

${ }^{20}$ While this complete indifference below $T$ is an abstraction of reality, our results should be robust to alternative utility specifications that embody the notion of localized pressure. Evidently, the key force behind our results is that greater emphasis is placed on whether the target $(T)$ has been met, even if by the narrowest of margins, and far less on how far the target was missed by. In the language of utility function representations, this spells an alternating convexity and concavity, which we encapsulate as a step function. Therefore, we anticipate that (mild) relaxations will not alter the headline features of our results as long as concave and convex regions are juxtaposed together, an indispensable element in the representation of localized pressure.

${ }^{21}$ Colloquially speaking, this would be the pressure-ridden sentiment expressed as something along the lines of "I am dead anyway if I don't accomplish T"'.
} 
the trade-off -and consequently, the optimal choice of crime- implies that it must be important to consider the available "scale" of crime $(N)$ when studying the incentive to misbehave, an importance that the literature seems to have overlooked thus far. Of course, the arbitrarily large size of $N$ that we allowed in Propositions 1-2 is not realistic, and we impose an upper limit on $N$ going forward. We probe its consequences in the Sections that follow, after first providing an example that underscores the importance of skew-ness in our analysis.

\subsection{Example: Comparison with Becker (1968) Framework}

We end this section by illustrating that our focus on skew-ness we expounded in this Section is neither coincidental nor imposed, but rather that the dis-continuity of $v_{T}$ naturally calls for it. That is, while the dis-continuous shape of $v_{T}$, to some readers, may seem "unusual" enough to be capable of generating incentives to misbehave on its own even in a standard Becker framework with no reference to skew-ness, we show in the following example that this is not the case. In fact, when skew-ness is shut down, any form of risk-taking -including fraud-would never be optimal in the standard analysis of a cliffhanging situation.

Example 1. Let $B>0$, and consider a Becker agent with symmetric binomial martingales. In this case, $N^{*}=0$.

Proof. This is a straightforward modification of proof of Proposition 1, proven in the appendix.

The conclusion that $N^{*}=0$ means agents who are restricted to choose from symmetric gambles would rather choose to do nothing and face $u$ "as is". To provide some interpretation, note that $B>0$ implies that the agent is on the (piece-wise) concave domain of $v$, and hence perhaps it is not surprising that a Becker agent would not commit to gambles. For this reason, Becker (1968) makes a somewhat odd claim that all criminals must be risk-lovers. Our approach, on the other hand, is to expand the choice set to provide the agents with explicit opportunities to play skewed bets to deal with their desperation. And indeed, when given such opportunities, agents show strong demand for negative skewness, which we interpret as an unequivocal incentive to misbehave.

\section{A "Micro-economic Framework" for Fraud}

In reality, the choice of $(N, p)$ is not completely unfettered; for example, it is implausible to assume that agents are able to scale up the fraud scheme $(N)$ to such an arbitrarily high level. We therefore 
start this section by postulating some structure on the available choice of $(N, p)$, in particular, by placing an upper limit on $N$. Limiting $N$ opens the door to a number of actions because the agent can no longer nullify the trade-off between the "gain" and "precision" by levering up the scale of the fraud scheme and therefore, comparative statics on other relevant parameters now becomes meaningful. The analysis develops itself into a framework that resembles standard micro-economic analysis.

\subsection{The Choice Set of Crime: from Petty to Serious}

The choice of $(N, p)$ in our model represents a choice of crime. We make two realistic observations. [Observation 1] For any given $p$, the maximal scale $(N)$ one can choose must be finite, as is any choice set in reality. We denote the least upper bound for the given $p$ as $\bar{N}_{p}$. [Observation 2] Moreover, it is entirely plausible that this upper bound will be lowered as $p$ increases close to 1 $(p \approx 1)$, simply because it is harder to conceal crime of bigger scale than it is to conceal crime of negligible scale. For example, it is most likely harder to conceal a tax evasion of $\$ 1,000,000$ than it is to conceal tax evasion of $\$ 10$. We denote a generic upper bound that works for all $p$ as $\bar{N}$.

These two observations allow us to illustrate the choice of crime $(N, p)$ graphically as Figure 6 below. The shaded area represents available crime to choose from. $\bar{N}$ is the boundary over which no crime of such scale can exist, regardless of $p$. Figure 6 also shows that the available scale of crime $\left(\bar{N}_{p}\right)$ given $p$ is decreasing in $p$ (Observation 2, i.e., $\frac{\partial \bar{N}_{p}}{\partial p}<0$ ). This downward sloping boundary specifies the "efficient frontier" of available crime ${ }^{22}$.

Figure 6 allows us to interpret the two extreme ends of $\left\{M_{b}(p): p>\frac{1}{2}\right\}$. First, $\left\{M_{b}(p), p \approx 1\right\}$ represent "petty crime", because the available scale $(N)$ of crime is likely to be very limited at those levels of $p$, as is shown in Figure 6. These are, much like minor incursions of the speed limit, very likely to go unpunished $(p \approx 1)$ and are small in scale $(N \approx 0)$. And similarly, we can interpret the other extreme, $\left\{M_{b}(p), p \approx \frac{1}{2}\right\}$, as more "serious crime" as they can be committed at a larger scale, so long as the agent is willing to accept a higher chance of apprehension (lower $p$ ). Moreover, note that the law enforcement authorities must - if they were to allocate their resources rationallydevote more attention to detecting "serious crime", which would invariably increase the detection

\footnotetext{
${ }^{22}$ Going forward, this nomenclature will make more sense as we introduce Propositions that will reveal that the agents prefer to be on the 'northeast' corner on Figure 6, much like in standard microeconomic analysis.
} 


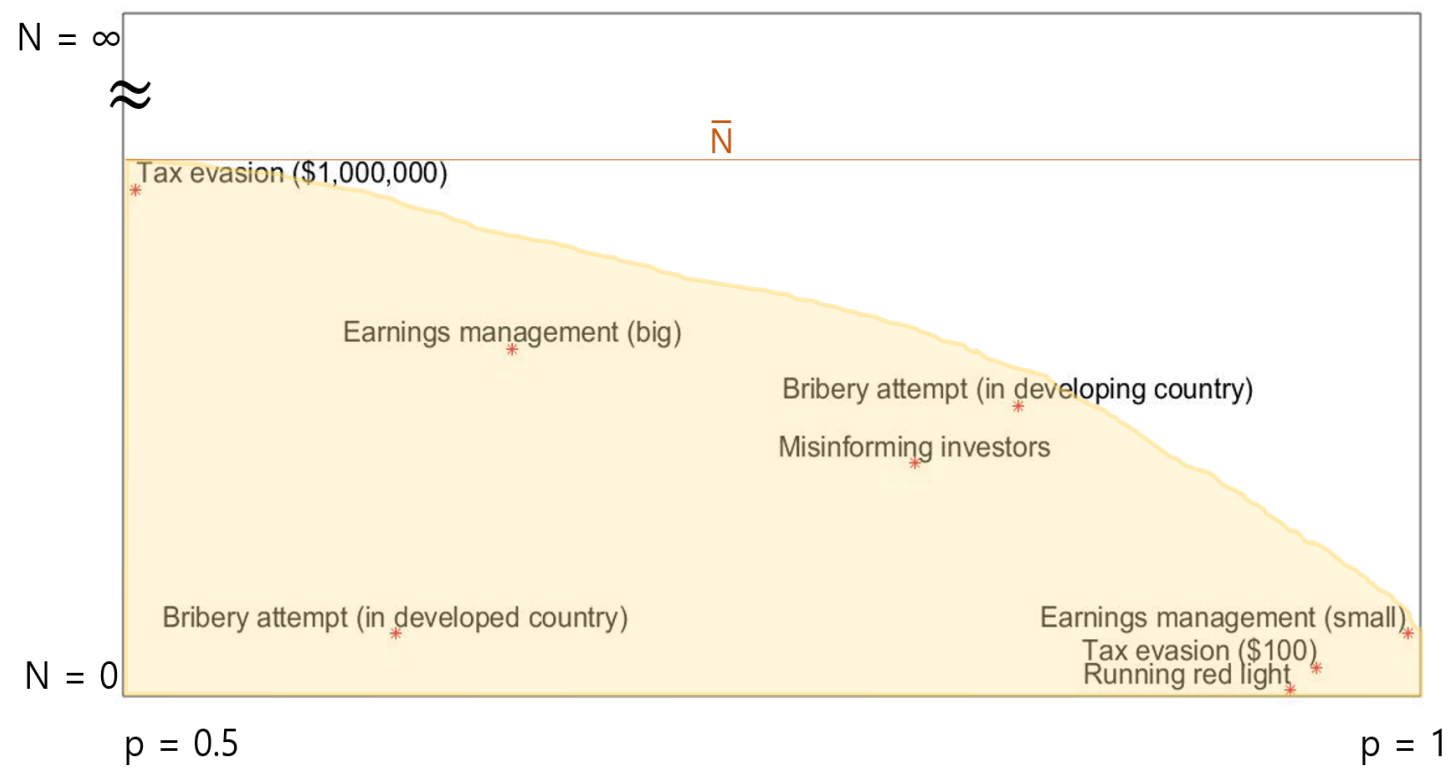

Figure 6: A Conceptual "Efficient Frontier" of Crime (based on observations 1 and 2)

rate. This, of course, leads to the conclusion that more serious crimes are more likely to get caught, providing an additional explanation for our interpretation that $p \approx \frac{1}{2}$ represents serious crime while $p \approx 1$ represents petty crime. With these in mind, we now explicitly consider the effects of placing a limit on $N$.

\subsection{The Preference Structure for Fraud}

Consider a uniform upper bound $\bar{N}$ in line with Observations 1 and 2. Also, we temporarily fix $B$ at 0 , and explore the consequences of varying $B$ separately in the section that follows.

Proposition 3 (Interior Solution). Let $B=0$. Let $\bar{N}<\infty$ be the upper limit to $N$. Then the solution is interior, i.e., $\exists p^{*} \in\left(\frac{1}{2}, 1\right)$.

Proof. See appendix.

Proposition 3 sets the stage for analysis with bounded $N$, by asserting that limiting $N$ delivers interior solutions. This allows for additional comparative statics analysis. Recall that in Proposition 2, the manager was able to keep increasing $\mathbb{E}\left[v_{T}\left(X^{\prime}(N, p)\right)\right]$ towards 0 -effectively eliminating the cliff (s)he faces- by driving $p$ arbitrarily close 1, essentially because $N$ was unrestricted. Proposition 3 states that this unrealistic maneuver is no longer available when $N$ is limited, and agents must 
choose an interior solution with $p^{*}<1$. This echoes our earlier interpretation of Proposition 2: that $p$ and $N$ are effectively complements. Namely, when $\mathrm{N}$ is limited, $N$ can no longer complement a very large choice of $p(p \approx 1)$, and agents are hurt by choosing a level of $p$ that cannot be supported by a commensurately high $N$. Therefore, $p^{*}<1$, which in turn means that a "corner solution" in Figure 6 will never be chosen.

On a slightly more technical note, this also ties up a loose end that we left behind: (4) was 'ill-posed' because agents could always do better by encroaching to infinitely large values of $N$, whereas $V_{\bar{N}}^{*}$ in the following restatement of (4) is now well-defined.

$$
V_{\bar{N}}^{*}:=\max _{\substack{p \in(0,1) \\ N \in[0, \bar{N}]}} \mathbb{E}\left[v_{T}\left(X^{\prime}(N, p)\right)\right]
$$

Mathematically, the only difference is that the domain is now bounded $(N \in[0, \bar{N}])$ and compact $^{23}$ making the problem well-defined.

Given the assertion that the solution is interior, the following Proposition makes explicit statements on the preference for fraud.

Proposition 4 (Pecking Order). Let $B=0$. Let $\bar{N}<\infty$ be the upper limit to $N$. Then

(i) [Monotonicity] $\bar{N}$ is always binding.

(ii) [Complementarity] $\exists \tilde{N}<\infty$ such that $\frac{\partial p^{*}}{\partial N}>0, \forall N>\tilde{N}$.

Proof. See appendix.

Recall the trade-off between "gain" and "precision" in Proposition 2, which can be alleviated by "levering up" the size of the fraud scheme $(N)$. Proposition 4- $(i)$ affirms this interpretation by asserting that $\bar{N}$ will always be depleted, simply because it helps override the trade-off. Furthermore, Proposition 4-(ii) explicitly shows that $p$ and $N$ are complements, also in line with our interpretation of Proposition 2. That is, since the act of "levering up" effectively makes the pursuit of negative skewness more attractive by loosening the grip of the trade-off therein, it leads to a choice of a higher $p$, thereby generating the complementarity between $N$ and $p$. Lastly, the qualification that Proposition 4-(ii) only works uniformly for $N>\tilde{N}$ simply represents the fact that the size of the bet must be large enough to help overcome the uncertainty $u$, otherwise there would be

\footnotetext{
${ }^{23}$ The domain of $p$ can also be easily compactified by adjoining the endpoints. And it is easy to see that doing so would not alter the solution, as Proposition 3 asserts that the solution is interior.
} 
no point in taking the risk. ${ }^{24}$ Hence, if the martingale gamble is very small, then the more likely outcome is simply to choose $N^{*}=0$ and accept the cliffhanging situation as it is.

It is worth noting that Proposition 4 implies a resemblance to indifference curves in standard micro-economics. Indeed, according to Proposition 4- $(i i)$, a loose restriction on $N$ leads to higher $p^{*}$, and consequently, higher $\mathbb{E}\left[v_{T}\left(X^{\prime}\right)\right]$ by Proposition 2-(ii). On the contrary, if the scale $(N)$ of the available fraud scheme is limited, the misbehavior must get bolder and riskier (i.e., lower $p^{*}$ ) to compensate for this limitation, much to the dismay of the agent as it leads to lower levels of $\mathbb{E}\left[v_{T}\left(X^{\prime}\right)\right]$. Thus, $N$ is always depleted (Proposition $4-(i)$ ). Visually, this means that agents will unequivocally prefer to be on the 'northeast' corner of Figure 7, much akin to the monotonicity of goods we assume in microeconomic analysis. Moreover, the complementarity of $p$ and $N$ (Proposition 4-(ii)) implies that the "indifference curves" are convex from below, as is also depicted in the blue curves of Figure 7. And by Proposition 3, we are reassured that an interior solution must exist within $p^{*}<1$.

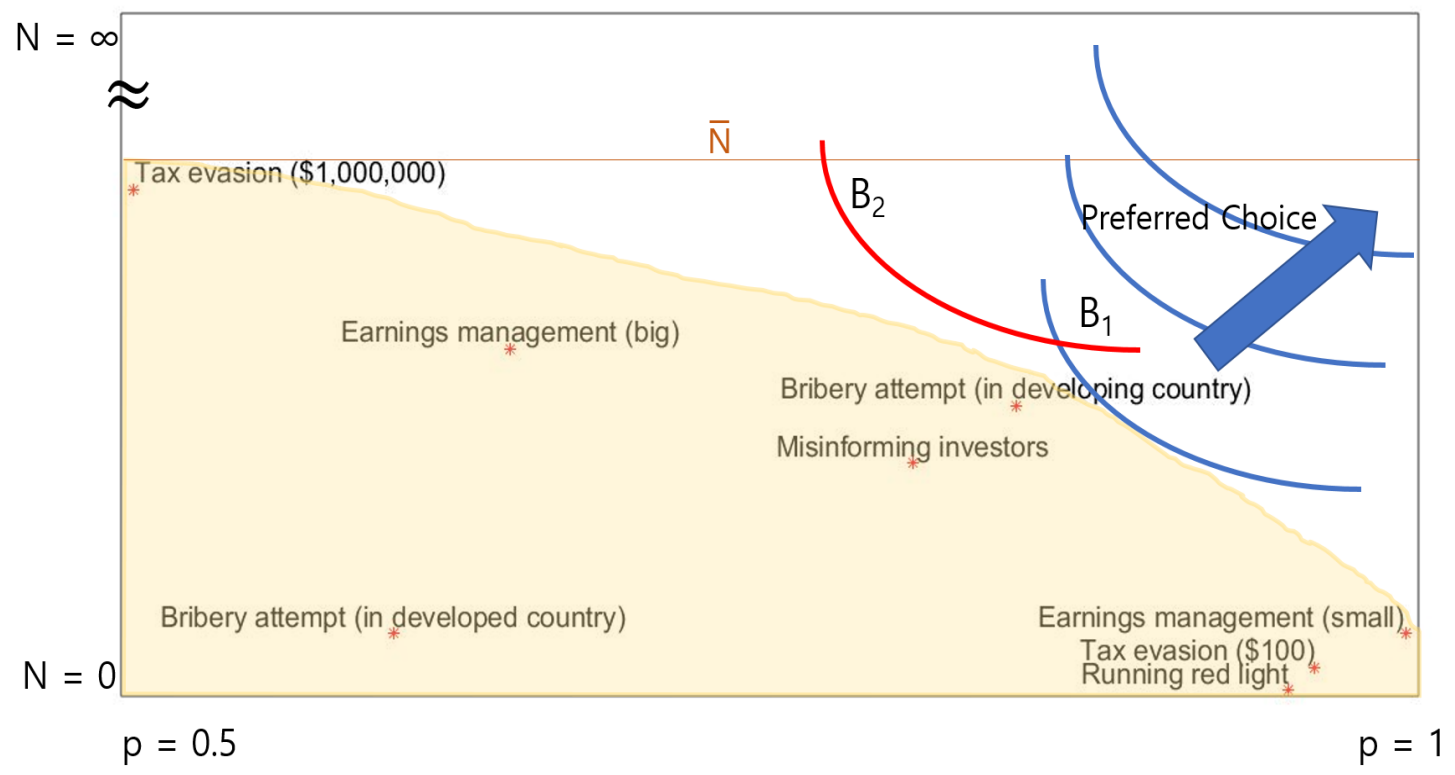

Figure 7: The Preference and Choice of Fraud

In the language of fraud, these findings allude to a 'pecking order', whereby the preference is to

\footnotetext{
${ }^{24}$ The qualifications on $N$ that appear in the Propositions that follow all share the same spirit, hence we do not repeat the same argument going forward.
} 
deplete the petty fraudulent choices first, and if such options are no longer available, resort to the riskier and more serious alternatives rather reluctantly. But insofar as Observation 2 holds, the choice set is concentrated around the 'southwest' corner of Figure 7, and the optimal choice $\left(N^{*}, p^{*}\right)$ would have to represent a balance between the two, as in any standard micro-economic analysis with a budget constraint and indifference curves. This 'pecking order' also explains the empirical findings in the literature that are often interpreted as managers' reluctance to resort to serious infringement of law until they exhaust the more legitimate and undetectable options. (See, for example, Beneish (1997) and Dechow et al., (2011).) Proposition 4 is also agrees with findings that estimate a prevalence of "low-cost" crimes -for example, $60 \%$ of CEOs engage in earnings misstatement (Zakolyukina, 2017).

\section{Some Comparative Statics: The Role of $B$}

In Propositions 3 - 4, we fixed $B=0$ for the sake of simplicity. However, insofar as $B$ specifies the initial distance from $T$ which the agent is acutely interested in exceeding, it is very probable that changing $B$ will alter the optimal choice. We explore this possibility in the next two Propositions. We continue to limit $N$ uniformly by $\bar{N}$. Then the optimal choice of skewness embodied in $p^{*}$, is a function of $\bar{N}$ and, presumably, also $B$. In particular, we denote the relationship between $p^{*}$ and $B$-fixing a specific value of $\bar{N}$ and all other parameters- $\operatorname{simply}$ as $p^{*}(B ; \bar{N}, \cdots)$.

\subsection{The "Slippery Slope" to Fraud: $B \geq 0$}

Proposition 5 (Slippery Slope). Consider the case where $\bar{N}<\infty$. Let $B \geq 0$.

(i) $\exists \bar{B}$ such that $N^{*}=0, \forall B>\bar{B}$.

(ii) Assume "single crossing" property. Then $\exists \underline{N}>2 \sigma$, such that $p^{*}(B ; \bar{N}, \cdots)$ is monotone increasing in $B$ for any $\bar{N} \in[\underline{N}, \infty)$.

Proof. See appendix.

Proposition 5- $(i)$ asserts that agents do not commit fraud when they feel that they are a "safe distance away from the cliff". Namely, there is a threshold value of $B$ after which commission of fraud is no longer attractive. This makes good sense, considering the fact that $v$ would asymptotically approach a flat line as $B \rightarrow \infty$. Proposition 5-(ii) states that the further away the agent stands from the cliff, the more negatively skewed bets (s)he prefers. While this may seem counter-intuitive 
because it means that those who are "safer" seem to prefer a more extreme form of skewness, this can also be easily understood within the framework of the trade-off between "gain" and "precision". When the agent perceives the cliff a safe distance away, the demand for "gain" dissipates, hence the agent would optimally choose to increase "precision" by choosing a higher $p$. The optimal fraud would thus be the safer and pettier fraud. Taken together, $(i)$ and $(i i)$ together tell us what happens when agents move incrementally further away from the cliff: at first, when $B=0$, agents resort to the most serious of frauds, then gradually they opt for safer and pettier frauds such as very modest earnings managements, after which they avoid commission of any type of unscrupulous act altogether.

It is interesting to note that Proposition 5 provides a theoretical explanation for Schrand and Zechman's empirical findings (2012) that CEO's overconfidence, which is often innocent in the beginning, compels them to start down a "slippery slope" that snowballs into serious misbehavior once the optimistic bias begins to give way to reality. ${ }^{25}$ An initial optimistic bias, in our model parlance means that the CEO perceives $X^{\prime}-T$ as being unreasonably large, and hence leads to an unrealistically high level of $B$. As the hard reality sets in, the perceived magnitude of $B$ shrinks. And by Proposition 5-(ii), this leads to lower $p^{*}$, coercing the CEO to ponder commission of more serious crime, who eventually starts down the "slippery slope" to misbehavior as $B$ ultimately corrects itself to 0. With our micro-economic framework, graphically described in Figure 7, this is expressed as a shift of indifference curves with $B_{1}$ to $B_{2}, \quad\left(B_{1}>B_{2}\right)$ whence the optimal choice of $p$ would presumably be lowered $\left(p_{1}^{*}>p_{2}^{*}\right)$, representing the glide down the "slippery slope" to increasingly more serious commissions of misbehavior.

\subsection{The Aspirational Case $(B<0)$}

Thus far, we have focused on the cliffhanging situation. The following Proposition considers an "aspirational" case where the agent is not on the cliff, but rather aspiring to jump above the cliff. This is worth exploring because aspirational setups -for example the convex payoff structure that typically sets an ambitious target to be attained such as in stock options- have often been named a possible source of a number of frictions, including fraudulent behavior. In our setup, this can be

\footnotetext{
${ }^{25}$ We quote directly from their abstract which reads: " $A$ detailed analysis of 49 firsm subject to AAERs suggest that ... the initial misstatement reflects an optimistic bias that is not necessarily intentional. Because of the bias, however, in subsequent periods these firms are more likely to be in a position in which they are compelled to intentionally misstate earnings. Overconfident executives are more likely to exhibit an optimistic bias and thus are more likely to start down a slippery slope of growing intentional misstatements..."
} 
modeled simply as $B<0$.

Proposition 6. Consider the case where $\bar{N}<\infty$ and $B<0$.

(i) $\exists \hat{N}$, such that for any $N>\hat{N}, \exists B<0$ where $p^{*}<\frac{1}{2}$ for the given parameters $(B, N)$

(ii) Proposition 5-(ii) extends to $B<0$. Namely, $\frac{p^{*}(B ; \bar{N}, \ldots)}{\partial B}>0$ holds under the same qualification even when $B<0$.

Proof. See appendix.

Proposition 6- $(i)$ reverts the conclusion of Proposition 1 which stated that $p^{*}>\frac{1}{2}$ must always hold when $B \geq 0$. In the aspirational case $(B<0)$, there are instances where positive skewness $\left(p<\frac{1}{2}\right)$ may be the optimal choice instead. Proposition 6-(ii) provides a hint as to when this is most likely to happen: when $B \ll 0$, namely when the aspiration is too remote to be attained realistically. This can, again, be easily understood by the trade-off between "gain" and precision. Suppose an agent is tantalizingly close to, but below $T$, i.e., $0 \approx B<0$. In this case, the agent would just need a small push forward to attain her aspiration, so the desired "gain" is rather small, allowing her to invest in "precision" instead, whence the likely choice is still negatively skewed $\left(p^{*}>\frac{1}{2}\right)$. However, as $B$ decreases further there is fewer hope of attaining $T$ unless the "gain" is engineered to be much larger at the expense of lowered $p$, until at some point it reaches $p^{*}=\frac{1}{2}$. Further decreasing $B$ (i.e., $B \ll 0$ ) would mean that her aspirations devolve down to an attempt of lottery, in which case it is not surprising that $p^{*} \approx 0$.

Figure 8 provides a pictorial summary of Proposition 6. The red regions (Regions B \& C) depict the zone where $\left\{M_{b}(p), p>\frac{1}{2}\right\}$ is optimal: the area where our model predicts white-collar crime is likely to be seen. The gradation denotes the severity of crime, where the severe ones $\left(p \approx \frac{1}{2}\right)$ are most likely to be concentrated around $X=T$ (where $B=0$ ). The prediction on severity notwithstanding, this does not mean that the model predicts a more frequent commission of white-collar crime around $X=T$. Quite the contrary, there are a number of reasons to believe that there will be more frequent commission of crime in the region with $B>0$ (Region $\mathrm{B}$ ) of our model than in the $B \approx 0$ region (Region $\mathrm{C}$ ). First, as per our "pecking order" result, the preference would be to deplete the petty frauds before they resort to the more serious alternatives as the situation deteriorates, thus on average, we would see more frequent commission of petty crime in Region B $(B \gg 0)$. Also, many of the gambles typically chosen in Region C (i.e., where the typical choice would be $\left\{M_{b}\left(p^{*}\right), p^{*} \approx \frac{1}{2}\right\}$, under $B \approx 0$ ) will actually represent other form of risk-taking, like 


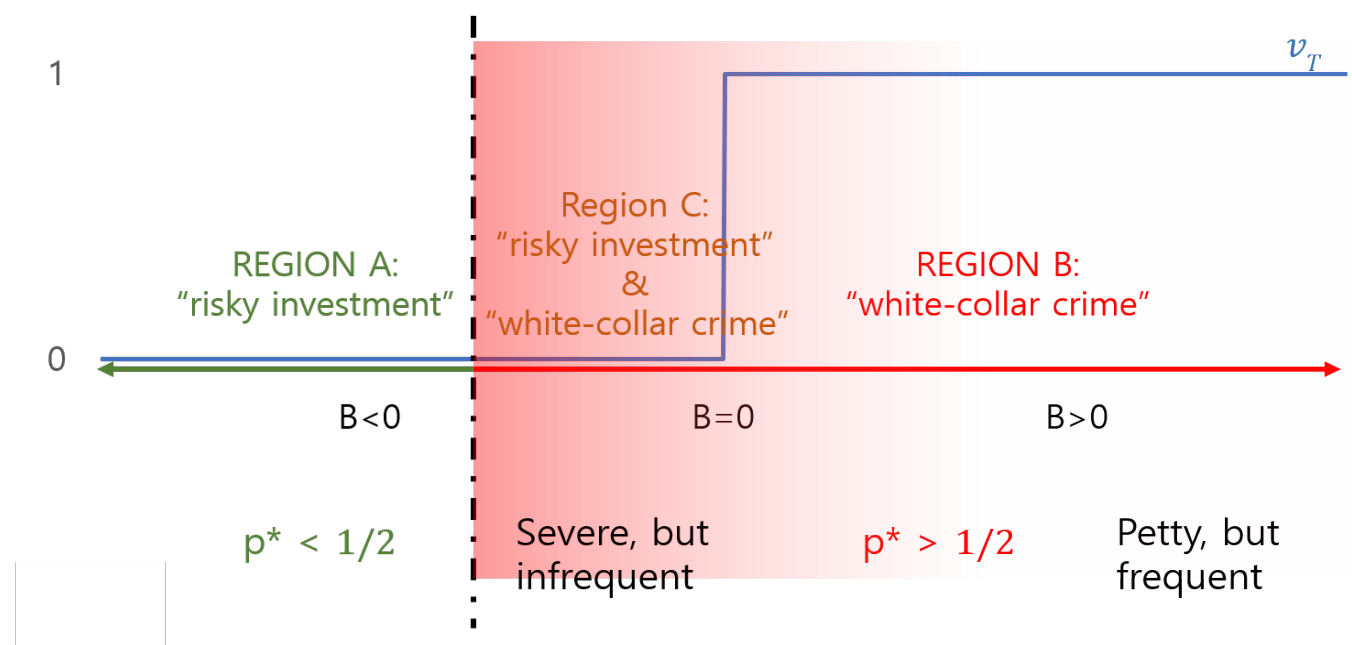

Figure 8: A graphical summary. Gradation represents seriousness of crime, not likelihood.

risky investments made by CEOs. Indeed, insisting that Region $\mathrm{C}$ (i.e., the region where agents typically choose $\left.p^{*} \approx \frac{1}{2}\right\}$ ) purely consist of criminal activities would stand squarely at odds with the the unanimous estimates in the literature that typically find much lower probabilities of detection, i.e., $p \gg \frac{1}{2}$ and hence typically occurring outside Region C.

Our interpretation of Regions A \& C as consisting of "risky investments" is, in part, motivated by the thick literature on CEO compensation that explores how convex payoff structure induces risktaking behavior of firm managers, sometimes at the expense of firm value. The setup in Region A is indeed very similar to the convex setup that is typical in the literature, where our model predicts demand for positively skewed gambles ${ }^{26}$, which translates directly to 'risky $\left(p^{*}<\frac{1}{2}\right)$ investments' in the CEO compensation literature. In this sense, the dashed vertical line that marks the boundary between $\left\{M_{b}\left(p^{*}\right), p^{*}<\frac{1}{2}\right\}$ and $\left\{M_{b}\left(p^{*}\right), p^{*}>\frac{1}{2}\right\}$ is probably where our model dissolves and mixes with the compensation literature, but with a clearly distinct perspective. The risk-taking channel in the compensation literature typically focuses on the second moment; namely the idea is that to attain an ambitious goal one must increase the variance (or risk) in order to assign some probability mass to meet or exceed the goal. Our framework provides a novel third moment perspective, which we believe is at least as plausible: the agent makes a tailored attempt to attain the aspiration -in the form or risky $\left(p^{*}<\frac{1}{2}\right)$ investments- by pushing the peak backwards and thickening the upper

\footnotetext{
${ }^{26}$ Aristdou et al. (2021) explores how aspiration - or managerial ambition in this setup - can lead to risk-taking in the form of seeking positive skew-ness.
} 
tail through positively skewed bets ${ }^{27}$.

\section{Empirical Predictions}

We gather some empirical predictions from our model, compare with findings in the literature, and suggest possible directions for further empirical investigations. Overall, by design, our model favors empirical results that revolve around the notion of desperation rather than aspiration. That is to say, in line with Proposition 6, we anticipate that the larger bulk of fraudulent behavior will occur when $B$ is positive or small (Regions $\mathrm{B} \& \mathrm{C}$ ), than when $B$ is negative (Region $\mathrm{A}$ ). There has been no study that directly tests for this conjecture, to the best of our knowledge. However, surveys of the literature (for example, Amiram et al., 2018) are largely supportive of our model in that they find very mixed results on whether stock-based compensation schemes (e.g., stock options, stock appreciation rights) trigger financial fraud, whereas empirical evidence for pressure-based stories are much stronger ${ }^{28}$. To the extent that stock-based compensations are innately aspirational $(B<0)$, -i.e., it would be self-contradictory to offer targets that have already been attained- whereas the distress-based rationale very much resonates with our focus on staying above the cliff $(B>0)$, we interpret the current overall standing of the literature as being strongly in support of our framework.

Our model also predicts that a gradually diminishing $B$ from above $(B>0)$ will engender incentive for agents to choose increasingly stronger forms of fraud schemes (decreasing $p^{*}$ ), in line with the notion of a "slippery slope". There are multiple situations where an agents may perceive $B$ to be decreasing, and hence feel compelled to "stick their necks out" by committing more serious fraud. One is -as Schrand and Zechman (2012) suggest and find some empirical evidence for- an initial optimism of the manager that gradually adjusts to reality. Another is a consistent record of beating analyst expectation, whence the detriment of missing the target is perceived to be increasingly more vivid and pungent. Chu et al. (2019) find evidence of such a build-up of pressure leading to earn-

\footnotetext{
${ }^{27}$ Note that this scheme works as a mirror reflection of the incentive to misbehave, which we explored in the previous section. The difference stems from the fact that we are deep in the aspirational case $(B \ll 0)$ and hence, positive skewness is optimal.

${ }^{28}$ Beneish (1999a) finds that manipulating firms face deteriorating gross margins, which he interprets as supportive of the distress/pressure narrative. Other findings in similar spirit include those by Johnson et al (2009) who report that firms experience decelerating growth in earnings per share before engaging in financial misconduct, Dechow et al. (2011), Chu et al. (2019), Schilit (2010) and Richardson et al. (2003), among many others. Amiram et al. (2018) concludes that "the combined evidence suggests that manipulators tend to be firms that have experienced strong past operating performance that is beginning to wane. The strong operating performance has led to elevated stock prices, and managers manipulate earnings in an effort to preserve their high stock prices", which is exactly what our framework intends to capture.
} 
ings manipulation, namely, that executives "initially engage in earnings management techniques that are within GAAP, but as expectations rise, executives use increasingly aggressive techniques". We predict that other situations where $B$ is perceived to decrease -for example, enlarging the magnitude of the drop, since it is topologically equivalent to bringing $B$ closer to the cliff- will lead to similar outcomes.

Perhaps the most challenging set of implications to test empirically is our prediction on the frequency and severity of financial misconduct. As per Proposition 4, we expect the petty and less detectable crime to be depleted first, and that they are likely to be pervasive. The empirical challenge here is obvious; because these predictions revolve around undetected fraud, which by definition is unobservable, it is difficult to gauge its magnitude and frequency. There have been recent attempts to fathom the unobservable; for example, Dyck et al. (2020), Wang (2013), Zakolyukina (2017) and Soltes (2019), whose findings are generally corroborative of our predictions. For example, Soltes (2019) states that "by examining internal company records... I show that misconduct is considerably more common -by several orders of magnitude - than indicated in any publically accessible data on corporate offending... (although they are) less egregious in nature (than the detected counterpart)..." Also, as noted in a previous section, there are empirical findings (Beneish (1997) and Dechow et al., (2011)) that support the notion that managers prefer to deplete the petty and safer misbehaviors before then engage in the more serious alternatives. Finally, by Proposition 6, we expect that -while the frequency may be relatively small- crime committed at around $B=0$ (Region C or somewhere along the boundaries of Regions B \& C) to be more severe and detrimental compared to those in $B>0$. If this is indeed born out to be true empirically, this may mean that it could be beneficial to concentrate enforcement around $B \approx 0$ from a policy perspective, even though the large bulk of "petty" criminal activity is being committed elsewhere, as Soltes (2019) seems to suggest.

\section{Conclusion}

We present a model of white-collar crime that neither relies on a strong belief in the first moment (positive expected payoff), nor a necessarily risk-seeking perpetrator, none of which is born out in empirical studies. Instead, we look at the interaction between "higher order (third) moment" considerations -i.e., negative skewness- and discontinuity in manager's payoffs, which are both 
empirically robust. We show how such a framework can be used to motivate and classify crime from the pettiest to the most serious. Despite minimalist assumptions, this also leads to a rich set of testable implications. The model also develops into a flexible "microeconomic" framework that is amenable to further theoretical applications.

Our model is clearly a simplistic abstraction of a much more complex reality, and is limited to that very extent. As a simplification, however, this makes it a stub of a framework that is amenable to realistic extensions. For example, a few obvious avenues include; adding additional dynamic structure as opposed to our trivially simplistic two period setup, relaxing the martingale assumption to super- (or sub-) martingales, exploring more realistic valuation functions $\left(v_{T}\right)$ such as those with positive slope, mollified or multiple discontinuities. As a "microeconomic" framework, the model is amenable to much of the analyses that is feasible in standard microeconomics, for example, the consequences of shrinking or expanding the choice set of crime in response to changes in law enforcement, or the business cycle. We envision that such analyses may lead to policy implications as well. 


\section{References}

[1] Amiram D., Z. Bozanic, J.D Cox, Q. Dupont, J.M. Karpoff, R. Sloan. (2018): "Financial Reporting and Other Forms of Misconduct: a Multidisciplinary Review of the Literature" Review of Accounting Studies, 23, 732-783.

[2] Amiram D., S. Huang, S. Rajopal. (2020): "Does Financial Reporting Misconduct Pay Off Even When Discovered?" Review of Accounting Studies, forthcoming.

[3] Aristidou A., A. Giga, S. Lee, F. Zapatero (2021): "Rolling the Skewed Die: An Economic Foundation for Demand for Skewness and Experimental Evidence". Working Paper.

[4] Arthaud-Day, M.L., Certo, S.T., Dalton, C.M., Dalton, D.R. (2006): "A Changing of Guard: Executive and Director Turnover During Corporate Financial Restatements" Academy of Management Journal, 49, 1119-1136.

[5] Bebchuk, L., Fried, J., Walker, D. (2002): "Managerial Power and Rent Extraction in the Design of Executive Compensation" University of Chicago Law Review, 69, 751-846.

[6] Becker, G.S. (1968): "Crime and Punishment: An Economic Approach.” Journal of Political Economy, 76, 169-217.

[7] Beneish, M.D. (1997): "Detecting GAAP Violation: Implications for Assessing Earnings Management Among Firms with Extreme Financial Performance" Journal of Accounting and Public Policy, 16 (3), 271-309.

[8] Beneish, M.D. (1999a): "The Detection of Earnings Manipulation” Financial Analysts Journal, $55(5), 24-36$.

[9] Bhojraj, S., P. Hribar, M. Picconi and J. McInnis (2009): "Making Sense of Cents: An Examination of Firms That Marginally Miss or Beat Analyst Forecasts" The Journal of Finance, 64 (5), 2361-2388.

[10] Burns, N. and S. Kedia (2006): "The Impact of Performance-based Compensation on Misreporting" Journal of Financial Economics, 79 (2006), 35-67.

[11] Chu, J., P. Dechow, K. Hui, and A. Wang. (2019): "Maintaining a Reputation for Consistently Beating Earnings Expectations and the Slippery Slope to Earnings Manipulation". Contemporary Accounting Research, 36 (4), 1966-1998. 
[12] Dechow, P.M., Ge, W., Larson, C.R., and Sloan, R.G. (2011): "Predicting Material Accounting Misstatements". Contemporary Accounting Research, 28 (1), 17-82.

[13] Desai, H., Hogan, C.E., and Wilkins, M.S., (2006): “The Reputational Penalty for Aggressive Accounting: Earnings Restatements and Management Turnover". The Accounting Review, 81 (1), 83-112.

[14] Dichev, I., J. Graham, C. Harvey, and S. Rajgopal (2013): "Earnings Quality from the Field". Journal of Accounting and Economics, 56, 1-33.

[15] Dyck, I,J,A., A. Morse, L. Zingales (2020): "How Pervasive is Corporate Fraud?" Working Paper.

[16] Feng, M., Ge, W. Luo, S., Shevlin, T. (2011): "Why Do CFOs Become Involved in Material Accounting Manipulations?" Journal of Accounting and Economics, 51, 21-36.

[17] Graham, J., C. Harvey, and S. Rajgopal (2005): "The Economic Implications of Corporate Financial Reporting" Journal of Accounting and Economics, 40, 3-73.

[18] Hennes, K., Leone, A., and Miller, B. (2008): "The Importance of Distinguishing Errors from Irregularities in Restatement Research: The Case of Restatements and CEO/CFO Turnover." The Accounting Review, 83 (6): 1487-1519.

[19] Jensen, M. C. (2005): "Agency Costs of Overvalued Equity." Financial Management, Spring 2005.

[20] Johnson, S.A., Ryan, H.E., and Tian, Y.S. (2009): "Managerial Incentives and Corporate Fraud: The Sources of Incentives Matter." Review of Finance, 13 (1): 115-145.

[21] Eisinger, J., (2017): The Chicken Club: Why the Justice Department Fails to Prosecute Executives. Simon \& Schuster. 2017.

[22] Karpoff, J.M., Lee, D.S., and Martin, G.S. (2008): "The Consequences to Managers for Financial Misrepresentation".

[23] Malmendier, U., and G. Tate (2005): "CEO Overconfidence and Corporate Investment". The Journal of Finance, 60 (6): 2661-2700. 
[24] Richardson, S., Tuna, I., Wu, M., (2003). "Predicting Earnings Management: the Case of Earnings Restatements." Unpublished working paper. University of Pennsylvania and HKUST.

[25] Schilit, H., (2010): Financial Shenanigans: How to Detect Accounting Gimmicks and Fraud in Financial Reports (p.318). New York: McGraw-Hill.

[26] Schrand, C., and S. Zechman. (2012): "Executive Overconfidence and the Slippery Slope to Financial Misreporting". Journal of Accounting and Economics, 53 (1): 311-329.

[27] Shapira, R., and L. Zingales (2017): Is Pollution Value-Maximizing? The DuPont Case. Working paper.

[28] Soltes, E. (2016): Why They Do It: Inside the Mind of the White-Collar Criminal. New York, Public Affairs Books.

[29] Soltes, E. (2019): "The Frequency of Corporate Misconduct: Public Enforcement Versus Private Reality". Journal of Financial Crime, 26 (4): 923-937.

[30] Wang, T.Y. (2013): "Corporate Securities Fraud: Insights from a New Empirical Framework." The Journal of Law, Economics, and Organization, 29 (3): 535-568.

[31] Zakolyukina, A. (2018): "How Common Are International GAAP Violations? Estimates from a Dynamic Model." Journal of Accounting Research, 56 (1): 5-44. 


\section{Appendix}

\section{A Proofs}

\section{Proof of Proposition 1}

Proof. Fix any $p$ and consider $\frac{\partial V(N, p)}{\partial N}$. Under our setup,

$$
V(N, p):=-\left((1-p) F_{L}(0 \mid B-N p)+p F_{U}(0 \mid B+N(1-p))\right), \quad \forall B \geq 0,
$$

hence,

$$
\begin{aligned}
\frac{\partial V}{\partial N} & =-\left((1-p) \frac{\partial F_{L}(0 \mid B-N p)}{\partial N}+p \frac{\partial F_{U}(0 \mid B+N(1-p))}{\partial N}\right) \\
& =p(1-p)\left(\phi_{L}(0 \mid B-N p)-\phi_{U}(0 \mid B+N(1-p)), \quad \forall B \geq 0,\right.
\end{aligned}
$$

where $F_{L}$ is the cumulative distribution function $(c d f)$ evaluated at 0 (the position of the cliff) conditional on the lower node realization of the binomial martingale, $F_{U}$ is the $c d f$ evaluated at 0 conditional on the upper node realization of the binomial martingale, and $\phi_{L}, \phi_{U}$ are the corresponding probability density functions $(p d f \mathrm{~s})$. Here, one can easily verify ${ }^{29}$ that

$$
\frac{\partial V}{\partial N} \leq 0, \quad \forall p \in\left(0, \frac{1}{2}\right], \quad \forall B \geq 0
$$

Thus $\frac{\partial V}{\partial N} \leq 0$ on $p \in\left(0, \frac{1}{2}\right]$ means $N=0$ is optimal whenever $p \in\left(0, \frac{1}{2}\right]$. This means no binomial martingale is bought, and agents stay with the initial allocation. Thus, any such $(0, p)$ profile would deliver identical expected utility regardless of $p$, since $N=0$ means the choice of binomial martingale is irrelevant. In particular, we just fix $p=\frac{1}{2}$ going forward.

Henceforth, it is enough to show that

$$
V\left(0, \frac{1}{2}\right)<V(N, p), \quad \forall B \geq 0
$$

for some $p>\frac{1}{2}$ and $N>0$. For this, consider an incremental increase of $(N, p)$ from $\left(0, \frac{1}{2}\right)$ to $\left(\frac{1}{\epsilon}, \frac{1}{2}+\epsilon\right)$, essentially finding the directional derivative ${ }^{30}$ in the direction of $\left(\frac{1}{t}, t\right)$. Computing

\footnotetext{
${ }^{29}$ When $B=0$ it is clear. If $B>0$, note that this is equivalent to the the point of evaluation ' 0 ' translated by $-B$. The inequality follows immediately from single peaked-ness.

${ }^{30}$ For our purpose, this is good enough, for we only need to show that there exists some choice that dominates $V\left(0, \frac{1}{2}\right)$.
} 


$$
\begin{aligned}
& \left.\Delta V\right|_{\left\{\left(0, \frac{1}{2}\right)\right\}}:=V\left(\frac{1}{\epsilon}, \frac{1}{2}+\epsilon\right)-V\left(0, \frac{1}{2}\right) \text { yields: } \\
& \left.\quad \Delta V\right|_{\left\{\left(0, \frac{1}{2}\right)\right\}}=\frac{1}{2}-\left(\left(\frac{1}{2}-\epsilon\right) F_{L}\left(0 \mid B-\frac{1}{\epsilon}\left(\frac{1}{2}+\epsilon\right)\right)+\left(\frac{1}{2}+\epsilon\right) F_{U}\left(0 \mid B+\frac{1}{\epsilon}\left(\frac{1}{2}-\epsilon\right)\right)\right), \quad \forall B \geq 0 .
\end{aligned}
$$

The task, then, is to sign (6).

(i) Consider first $B=0$. Rearrangining (6) under $B=0$,

$$
\begin{aligned}
\left.\Delta V\right|_{\left\{\left(0, \frac{1}{2}\right)\right\}=} & \frac{1}{2}-\left(\left(\frac{1}{2}-\epsilon\right) F_{L}\left(0 \mid-\frac{1}{2 \epsilon}-1\right)+\left(\frac{1}{2}+\epsilon\right) F_{U}\left(0 \mid \frac{1}{2 \epsilon}-1\right)\right) \\
= & \frac{1}{2}-\left(\left(\frac{1}{2}-\epsilon\right) F_{L}\left(1+\frac{1}{2 \epsilon} \mid 0\right)+\left(\frac{1}{2}+\epsilon\right) F_{U}\left(1-\frac{1}{2 \epsilon} \mid 0\right)\right) \\
= & \frac{1}{2}-\left(\left(\frac{1}{2}-\epsilon\right)\left(1-\mathbb{P}\left(u>1+\frac{1}{2 \epsilon} \mid 0\right)\right)+\left(\frac{1}{2}+\epsilon\right) \cdot \mathbb{P}\left(u \leq 1-\frac{1}{2 \epsilon} \mid 0\right)\right) \\
= & \frac{1}{2}-\left(\left(\frac{1}{2}-\epsilon\right)\left(1-\mathbb{P}\left(u \leq-1-\frac{1}{2 \epsilon} \mid 0\right)\right)\right. \\
& \left.+\left(\frac{1}{2}+\epsilon\right)\left(\mathbb{P}\left(u \leq-1-\frac{1}{2 \epsilon} \mid 0\right)+\mathbb{P}\left(-1-\frac{1}{2 \epsilon} \leq u \leq 1-\frac{1}{2 \epsilon} \mid 0\right)\right)\right) \\
= & \frac{1}{2}-\left(\left(\frac{1}{2}-\epsilon\right)+2 \epsilon \cdot \mathbb{P}\left(u \leq-1-\frac{1}{2 \epsilon} \mid 0\right)+\left(\frac{1}{2}+\epsilon\right) \cdot \mathbb{P}\left(-1-\frac{1}{2 \epsilon} \leq u \leq 1-\frac{1}{2 \epsilon} \mid 0\right)\right) \\
> & \epsilon-2 \epsilon \cdot \mathbb{P}\left(u \leq-1-\frac{1}{2 \epsilon} \mid 0\right)-\left(\frac{1}{2}+\epsilon\right) \cdot \mathbb{P}\left(-1-\frac{1}{2 \epsilon} \leq u \leq 1-\frac{1}{2 \epsilon} \mid 0\right) \\
> & \epsilon-\epsilon \frac{\sigma^{2}}{\left(1+\frac{1}{2 \epsilon}\right)^{2}}-\left(\frac{1}{2}+\epsilon\right) \cdot \frac{\sigma^{2}}{\left(-1+\frac{1}{2 \epsilon}\right)^{2}} \\
= & \epsilon-\phi\left(\epsilon^{3}\right) \\
> & 0,
\end{aligned}
$$

whenever $\epsilon>0$ is "small enough", given $\phi$, the 'little o' notation. The equalities are straightforward manipulations using symmetry and properties of $c d f$, and the second inequality is by Markov.

(ii) When $B>0$, it is easy to verify - using the logic of an earlier footnote and the non-decreasing property of $c d f$ - that $\left.\Delta V\right|_{\left\{\left(0, \frac{1}{2}\right)\right\}}$ is increasing in $B$. Hence, $\left.\Delta V\right|_{\left\{\left(0, \frac{1}{2}\right)\right\}}>0$, and therefore $p \notin\left(0, \frac{1}{2}\right]$ since agents can always do better by choosing $p>\frac{1}{2}$ instead of $p \leq \frac{1}{2}$. In fact, $V\left(0, \frac{1}{2}\right)$ represents a saddle point. Q.E.D.

\section{Proof of Proposition 2}

Proof. For notational tidiness, we prove only for $B=0$, but the proof for $B>0$ is almost identical. 
We also note that since we assume $u$ is a continuous random variable, its $c d f$ is differentiable.

Consider the approximation:

$$
\Delta V(N, p)=\frac{\partial V^{+}}{\partial p} \Delta p+\varnothing(\Delta p)
$$

where the ' + ' superscript denotes that the approximation is from 'above ${ }^{31}$ ' and $\varnothing$ is the 'little o' notation which in particular has the property:

$$
\lim _{\Delta p \rightarrow 0} \frac{\phi(\Delta p)}{\Delta p}=0
$$

The arbitrarily chosen (and fixed) monotone sequence $\left\{p_{k}\right\}_{0}^{\infty}$ also determines a sequence of increments: $\left\{\Delta p_{k}\right\}_{1}^{\infty}$. Our goal is to show that for each element $p_{k}$ there is a corresponding $N_{k}$ such that it renders $\Delta V\left(N_{k}, p_{k}\right)>0$. Showing that this can be done for any $k$ would conclude the proof.

In the current setup,

$$
V(N, p):=-\left((1-p) F_{L}(0 \mid-N p)+p F_{U}(0 \mid(1-p) N)\right) .
$$

Rewriting using symmetry,

$$
V(N, p):=-\left((1-p) F_{L}(N p \mid 0)+p F_{U}(-(1-p) N \mid 0)\right)
$$

Hence,

$$
\frac{\partial V(N, p)}{\partial p}=\left(F_{L}(N p \mid 0)-F_{U}(-(1-p) N \mid 0)\right)-N\left((1-p) F_{L}^{\prime}(N p \mid 0)+p F_{U}^{\prime}(-(1-p) N \mid 0)\right) .
$$

The direction we will pursue going forward is to show that for any given $p$ it is always possible, pointwise - i.e., for that particular given $p$, - to find a corresponding $N$ such that $\frac{\partial V}{\partial p}>0$. This is because the first big term can be made arbitrarily close to 1, and the second big term will be bounded by Markov inequality, hence $\frac{\partial V}{\partial p}$ can be made positive, essentially proving the Proposition.

\footnotetext{
${ }^{31}$ That is, this specifies a particular rule to choose the evaluation point on each term, given differentiability of $F$. On each term that constitute $\frac{\partial V}{\partial p}$ (clearly, in this setup, $\frac{\partial V}{\partial p}$ can be decomposed into separately differentiable terms), use the evaluation point $\hat{p}_{k} \in\left[p_{k}, p_{k+1}\right]$ which maximizes the particular term of the derivative. This choice of evaluation point is innocuous. For a 'sketch' of proof, consider a derivative with two terms so that the standard approximation becomes $\Delta V=\left(\left.a\right|_{p_{k}}+\left.b\right|_{p_{k}}\right) \Delta p+\varnothing(\Delta p)$, which is to use the lower ends $p_{k}$ as evaluation points. Consider the alternative approximation from 'above', $\Delta \hat{V}:=\left(\left.a\right|_{\hat{p}_{a, k}}+\left.b\right|_{\hat{p}_{b, k}}\right) \Delta p+\varnothing(\Delta p)$, where $\hat{p}_{a, k}$ and $\hat{p}_{b, k} \in\left[p_{k}, p_{k+1}\right]$. By termwise differentiability (and the obvious fact that $\lim _{\Delta p_{k} \rightarrow 0} \hat{p}_{a, k}=\lim _{\Delta p_{k} \rightarrow 0} \hat{p}_{b, k}=p_{k}$ ), we know that $\left.\left.a\right|_{\hat{p}_{a, k}} \rightarrow a\right|_{p_{k}}$ and $\left.\left.b\right|_{\hat{p}_{b, k}} \rightarrow b\right|_{p_{k}}$. Hence, rewriting, $\left.a\right|_{\hat{p}_{a, k}} \Delta p_{k}=\left.a\right|_{p_{k}} \Delta p_{k}+\varnothing_{a}\left(\Delta p_{k}\right)$ and $\left.b\right|_{\hat{p}_{b, k}} \Delta p_{k}=\left.b\right|_{p_{k}} \Delta p_{k}+\emptyset_{b}\left(\Delta p_{k}\right)$. Plugging this back to the approximations and lumping the 'little o's yields $\Delta V=\Delta \hat{V}+\hat{\varnothing}(\Delta p)$. Hence, we can redefine evaluation points simply by switching the error function ( $\varnothing$ 's), crucially, with identical limit properties around 0 .
} 
However, since (7) is only an approximation, establishing $\frac{\partial V}{\partial p}>0$ alone is not completely enough to prove the Proposition. The approximation error, $\varnothing$, is essentially local, pertaining locally to each interval $\Delta p_{k}$. So we first regulate them in order to limit the error uniformly over the entire family of $\varnothing$ 's. On the given $\left\{p_{k}\right\}_{0}^{\infty}$, consider adjacent pairs $\Delta p_{k}$ 's, and let

$$
\Delta V_{k}:=\left.\frac{\partial V^{+}}{\partial p}\right|_{\Delta p_{k}} \Delta p_{k}+\emptyset_{k}\left(\Delta p_{k}\right)
$$

where $\emptyset_{k}$ is the 'little $o$ ' pertaining to the approximation of $V$ around $\Delta p_{k}$. Let

$$
\varnothing:=\sup _{k}\left\{\varnothing_{k}\right\}_{k=1}^{\infty}
$$

Note that

$$
\lim _{\Delta p \rightarrow 0} \frac{\varnothing(\Delta p)}{\Delta p}=0
$$

inheriting from the definition of supremum and 'little o'.

Pick $0<\epsilon<\frac{1}{3}$. From (11), $\exists k_{0}$ such that

$$
\left|\frac{\varnothing\left(\Delta p_{k}\right)}{\Delta p_{k}}\right|<\epsilon<\frac{1}{3}, \quad \forall k \geq k_{0} .
$$

Such a $k_{0}$ can be found because $\left\{p_{k}\right\}_{0}^{\infty}$ converges to 1 , and is therefore Cauchy so that the corresponding $\left\{\Delta p_{k}\right\}_{1}^{\infty}$ converges to 0 . By construction, $\epsilon$ will be the uniform upper bound for the errors, uniform over all $k>k_{0}$. We only prove monotonicity for $k \geq k_{0}$, since monotonicity for its (finite) complement is straightforward to show.

Consider again the approximation on any $k \geq k_{0}$

$$
\Delta V_{k}:=\left.\frac{\partial V(N, p)^{+}}{\partial p}\right|_{\Delta p_{k}} \Delta p_{k}+\varnothing\left(\Delta p_{k}\right),
$$

where

$$
\begin{aligned}
\left.\frac{\partial V(N, p)^{+}}{\partial p}\right|_{\Delta p_{k}}:= & \left(F_{L}\left(N p_{k+1} \mid 0\right)-F_{U}\left(-\left(1-p_{k}\right) N \mid 0\right)\right) \\
& -N\left(\left(1-p_{k+1}\right) F_{L}^{\prime}\left(N p_{k+1} \mid 0\right)+p_{k} F_{U}^{\prime}\left(-\left(1-p_{k}\right) N \mid 0\right)\right)
\end{aligned}
$$

Note that the choice of evaluation points $\left(\hat{p}_{k}\right)$ in the above equation was made by acknowledging the (increasing) property of the $c d f(F)$ as well as the assumption the $u$ is uni-modal and hence its 
$p d f$ is decreasing around the mean.

From definition of $c d f$ 's,

$$
\lim _{N \rightarrow \infty} F_{L}\left(N p_{k+1} \mid 0\right)-F_{U}\left(-\left(1-p_{k}\right) N \mid 0\right)=1,
$$

for any fixed $p_{k}$ and $p_{k+1}$. In particular, it is always possible to find $N_{1, k} \in \mathbb{N}$ (contingent on a fixed $k$ ) such that

$$
F_{L}\left(N_{1, k} \cdot p_{k+1} \mid 0\right)-F_{U}\left(-N_{1, k} \cdot\left(1-p_{k}\right) \mid 0\right)>\frac{2}{3} .
$$

Furthermore, we can bound the second term in (9) using the the following set of estimates:

$$
\begin{aligned}
N\left(\left(1-p_{k+1}\right) F_{L}^{\prime}\left(N p_{k+1} \mid 0\right)+\right. & \left.p_{k} F_{U}^{\prime}\left(-\left(1-p_{k}\right) N\right) \mid 0\right) \Delta p_{k} \\
& =\left(\left(1-p_{k+1}\right) \Phi\left(N p_{k+1} \mid 0\right) \Delta\left(N p_{k}\right)+p_{k} \Phi\left(N\left(1-p_{k}\right) \mid 0\right) \Delta\left(N p_{k}\right)\right) \\
& <\left(\left(1-p_{k+1}\right) \cdot \int_{N p_{k}}^{N p_{k+1}} \Phi(\xi \mid 0) d \xi+p_{k} \cdot \int_{N\left(1-p_{k+1}\right)}^{N\left(1-p_{k}\right)} \Phi(\xi \mid 0) d \xi\right) \\
& <\left(\left(1-p_{k+1}\right) \cdot \int_{N p_{k}}^{\infty} \Phi(\xi \mid 0) d \xi+p_{k} \cdot \int_{N\left(1-p_{k+1}\right)}^{\infty} \Phi(\xi \mid 0) d \xi\right) \\
& <\left(\left(1-p_{k+1}\right) \cdot \mathbb{P}\left(|u|>N p_{k}\right)+p_{k} \cdot \mathbb{P}\left(|u|>N\left(1-p_{k+1}\right)\right)\right) \\
& <\frac{\sigma^{2}}{N^{2}}\left(\frac{1-p_{k+1}}{p_{k}^{2}}+\frac{p_{k}}{\left(1-p_{k+1}\right)^{2}}\right)
\end{aligned}
$$

The first equality is mere rewriting, and the first inequality is a combination of mean value theorem for integrals and the fact that $F$ represents a uni-modal distribution. The remaining inequalities are straightforward manipulations, and the final inequality is from Markov inequality.

From the above inequality, it is always possible to find $N_{2, k} \in \mathbb{N}$ (contingent on a fixed $k$ ) such that:

$$
-N_{2, k}\left(\left(1-p_{k+1}\right) F_{L}^{\prime}\left(N_{2, k} \cdot p_{k+1} \mid 0\right)+p_{k} F_{U}^{\prime}\left(-N_{2, k} \cdot\left(1-p_{k}\right) \mid 0\right)\right) \Delta p_{k}>-\frac{1}{3}
$$

Let

$$
N_{k}:=\max \left\{N_{1, k}, N_{2, k}\right\}
$$

On any sequence $\left\{p_{k}\right\}_{0}^{\infty} \uparrow 1$, choosing the corresponding $\left\{N_{k}\right\}_{0}^{\infty}$ according to (16) will satisfy (14) and (15). And recall that we have already chosen $k_{0}$ such that (12) always holds.

Pulling all these together, consider the given the sequence $\left\{p_{k}\right\}_{0}^{\infty} \uparrow 1$ and the correspondingly 
chosen $\left\{N_{k}\right\}_{0}^{\infty}$. Then on any $k \geq k_{0},\left(N_{k}, p_{k}\right)$ will satisfy:

$$
\begin{aligned}
\Delta V_{k} & :=\left.\frac{\partial V^{+}}{\partial p}\right|_{\Delta p_{k}} \Delta p_{k}+\emptyset_{k}\left(\Delta p_{k}\right) \\
& =\left(F_{L}\left(N_{k} p_{k+1} \mid 0\right)-F_{U}\left(\left(1-p_{k}\right) N_{k} \mid 0\right)\right) \Delta p_{k} \\
& -N\left(\left(1-p_{k+1}\right) F_{L}^{\prime}\left(N p_{k+1} \mid 0\right)+p_{k} F_{U}^{\prime}\left(-\left(1-p_{k}\right) N\right) \mid 0\right) \Delta p_{k}+\emptyset_{k}\left(\Delta p_{k}\right) \\
& >\frac{2}{3} \Delta p_{k}-\frac{1}{3} \Delta p_{k}-\frac{1}{3} \Delta p_{k} \\
& >0
\end{aligned}
$$

as desired.

Lastly, we give an example where $V\left(N_{k}, p_{k}\right) \stackrel{k \rightarrow \infty}{\longrightarrow} 0$. For any $p_{k} \in\left\{p_{k}\right\}_{0}^{\infty}$, let $N_{k}=\frac{1}{\left(1-p_{k}\right)^{2}}$. Then

$$
\begin{aligned}
\lim _{k \rightarrow \infty} V\left(N_{k}, p_{k}\right) & =\lim _{k \rightarrow \infty}-\left(\left(1-p_{k}\right) F_{L}\left(0 \mid-\frac{1}{1-p_{k}}\right)+p_{k} F_{U}\left(0 \mid \frac{1}{1-p_{k}}\right)\right) \\
& =-(0 \cdot 1+1 \cdot 0)=0
\end{aligned}
$$

as desired (although monotonicity is not guaranteed.) Q.E.D.

\section{Proof of Proposition 3}

Proof. Consider an arbitrary $N \leq \bar{N}$. For any $B \geq 0$, denote:

$$
V(N, p ; B):=-((1-p) F(N p-B \mid 0)+p F(-(1-p) N-B \mid 0))
$$

and

$$
\Delta V_{p}:=\frac{\partial V(N, p ; B)}{\partial p} .
$$

Then, for $\epsilon \in\left[0, \frac{1}{2}\right]$, let $p=\frac{1}{2}+\epsilon$ and consider $^{32}$

$$
\begin{aligned}
\left.\Delta V_{p}\right|_{\left\{B=0, p=\frac{1}{2}+\epsilon\right\}}= & \left.\frac{\partial V(N, p ; B)}{\partial p}\right|_{\left\{B=0, p=\frac{1}{2}+\epsilon\right\}} \\
= & \left(F\left(\frac{1}{2} N+N \epsilon \mid 0\right)-F\left(-\frac{1}{2}+N \epsilon \mid 0\right)\right) \\
& \quad-N\left(\left(\frac{1}{2}-\epsilon\right) F^{\prime}\left(\frac{1}{2} N+N \epsilon \mid 0\right)+\left(\frac{1}{2}+\epsilon\right) F^{\prime}\left(-\frac{1}{2} N+N \epsilon \mid 0\right)\right) .
\end{aligned}
$$

Note that clearly, $(i) V(N, p ; B)$ is continuously differentiable, in particular, $\frac{\partial V}{\partial p}$ is continuous in both $p$ and $N$. We show the following. (ii) If $\epsilon=0$, then $\left.\Delta V_{p}\right|_{\left\{B=0, p=\frac{1}{2}\right\}}>0$, shown in Lemma

\footnotetext{
${ }^{32}$ From Proposition 1 , we know $p \in\left(\frac{1}{2}, 1\right]$ so we limit the solution space accordingly.
} 
1. (iii) If $\epsilon=\frac{1}{2}$, then $\left.\Delta V_{p}\right|_{\{B=0, p=1\}}<0$, shown in Lemma 2. Then from the intermediate value theorem (IVT), $\exists p^{*} \in\left(\frac{1}{2}, 1\right)$ such that $\frac{\partial V\left(N, p^{*}\right)}{\partial p}=0$.

Lemma 1. Fix $N \leq \bar{N}$ and let $B=0$. When $\epsilon=0,\left.\Delta V_{p}\right|_{\left\{B=0, p=\frac{1}{2}\right\}}>0$.

Proof. When $\epsilon=0$,

$$
\begin{aligned}
\left.\frac{\partial V(N, p ; B)}{\partial p}\right|_{\left\{B=0, p=\frac{1}{2}\right\}} & =\left(F\left(\frac{1}{2} N \mid 0\right)-F\left(-\frac{1}{2} N \mid 0\right)\right)-\frac{N}{2}\left(F^{\prime}\left(\frac{1}{2} N \mid 0\right)+F^{\prime}\left(-\frac{1}{2} N \mid 0\right)\right) \\
& >0,
\end{aligned}
$$

where the last inequality follows from symmetry and uni-modality.

Lemma 2. Fix $N \leq \bar{N}$ and let $B=0$. When $\epsilon=\frac{1}{2},\left.\Delta V_{p}\right|_{\{B=0, p=1\}}<0$.

Proof. When $\epsilon=\frac{1}{2}$,

$$
\begin{aligned}
\left.\frac{\partial V(N, p ; B)}{\partial p}\right|_{\left\{B=0, p=\frac{1}{2}\right\}} & =F(N \mid 0)-F(0 \mid 0)-N \cdot F^{\prime}(0 \mid 0) \\
& =\int_{0}^{N} F^{\prime}(x \mid 0) d x-\int_{0}^{N} F^{\prime}(0 \mid 0) d x \\
& <0
\end{aligned}
$$

where the last inequality follows from uni-modality.

And since these hold for any fixed $N \leq \bar{N}$, standard application of IVT completes the proof. Q.E.D.

\section{Proof of Proposition 4}

Proof. Consider the setup in the proof of Proposition 3:

$$
V(N, p ; B):=-((1-p) F(N p-B \mid 0)+p F(-(1-p) N-B \mid 0))
$$

and its associated $\Delta V_{p}:=\frac{\partial \Delta V_{p}}{\partial p}$.

(i) We start with a Lemma.

Lemma 3. Fix $N \leq \bar{N} .\left.\Delta V_{p}\right|_{\left\{B=\frac{N}{2}, p=1\right\}}>0$. 
Proof.

$$
\begin{aligned}
\left.\frac{\partial V(N, p ; B)}{\partial p}\right|_{\left\{B=\frac{N}{2}, p=1\right\}} & =\left(F\left(\frac{N}{2} \mid 0\right)-F\left(-\frac{1}{2} \mid 0\right)\right)-N \cdot F^{\prime}\left(-\frac{N}{2} \mid 0\right) \\
& >0
\end{aligned}
$$

where the last inequality follows from symmetry and uni-modality.

Furthermore, recall the conclusion of Lemma 2: $\left.\Delta V_{p}\right|_{\{B=0, p=1\}}<0$. By applying the intermediate value theorem on $\left.\Delta V_{p}(B ; N, \cdots)\right|_{\{p=1\}}$, viewed as a single variable function in $B$, we conclude that $\exists \bar{B} \in\left(0, \frac{N}{2}\right)$, such that $\left.\Delta V_{p}(\bar{B} ; N, \cdots)\right|_{\{p=1\}}=0$. It is straightforward to show that $\left.\frac{\partial \Delta V_{p}}{\partial p}\right|_{\{p=1\}}>0$, and hence $p^{*}=1$ when $B=\bar{B}$. Meanwhile, borrowing the conclusion of Proposition 5-(ii) below -which can be proved independently - we can further conclude that $p^{*}=1, \forall B \geq \bar{B}$. Finally, we note that, by construction, the choice of $p=1$ is equivalent to choosing $N=0$, which then completes the proof.

(ii) We proceed by first laying down arguments that hold irrespective of any fixed $N$ until we reach Lemma 8, where we then explicitly specify restrictions on $N(N>\underline{N})$. Note that by first order condition, $\Delta V_{p}\left(p^{*}, B ; N\right)=0$ must hold for all $p^{*}, B$ at any given $N$. In order to show that $p^{*}(B ; N, \cdots)$ is increasing in $B$ it suffices to show that the signs of $\frac{\partial \Delta V_{p}}{\partial p}$ and $\frac{\partial \Delta V_{p}}{\partial B}$ are always in the opposite direction, by dint of the implicit function theorem. Differentiating $V(N, p ; B)$ ) with respect to $p$ (or more precisely, with respect to $\epsilon$, from the assumed relationship $p=\frac{1}{2}+\epsilon$ ),

$$
\begin{aligned}
\left.\Delta V_{p}\right|_{\left\{p=\frac{1}{2}+\epsilon\right\}}= & \left.\frac{\partial V(N, p ; B)}{\partial \epsilon}\right|_{\left\{p=\frac{1}{2}+\epsilon\right\}} \\
= & \left(F\left(\frac{1}{2} N+N \epsilon-B \mid 0\right)-F\left(-\frac{1}{2}+N \epsilon-B \mid 0\right)\right) \\
& \quad-N\left(\left(\frac{1}{2}-\epsilon\right) F^{\prime}\left(\frac{1}{2} N+N \epsilon-B \mid 0\right)+\left(\frac{1}{2}+\epsilon\right) F^{\prime}\left(-\frac{1}{2} N+N \epsilon-B \mid 0\right)\right) .
\end{aligned}
$$

Further differentiation yields the following:

$$
\frac{\partial \Delta V_{p}}{\partial p}=\frac{\partial \Delta V_{p}}{\partial \epsilon}=N\left(2(f(U)-f(D))-N\left(\left(\frac{1}{2}-\epsilon\right) f^{\prime}(U)-\left(\frac{1}{2}-\epsilon\right) f^{\prime}(D)\right)\right),
$$

and similarly,

$$
\frac{\partial \Delta V_{p}}{\partial B}=\left(-(f(U)-f(D))+N\left(\left(\frac{1}{2}-\epsilon\right) f^{\prime}(U)-\left(\frac{1}{2}-\epsilon\right) f^{\prime}(D)\right)\right)
$$


where

$$
U:=\frac{N}{2}+N \epsilon-B
$$

and

$$
D:=-\frac{N}{2}+N \epsilon-B,
$$

and $f$ denotes the probability density (i.e., $F^{\prime}=f$ ). Unfortunately, neither $\frac{\partial \Delta V_{p}}{\partial p}$ nor $\frac{\partial \Delta V_{p}}{\partial B}$ are monotone in their arguments, hence it is not straightforward to sign them. So to proceed, we make a few observations first.

Fix all variables, other than $p$ and $B$, to an arbitrary number. We define some notations. Denote $\mathcal{C}$ as the values of $(p, B)$ where $B>\epsilon N$, (or equivalently, when $B>\left(p-\frac{1}{2}\right) N$,) holds. Denote $\mathcal{P}^{+}$, and $P^{-}$as sets of values of $(p, B)$ where $\frac{\partial \Delta V_{p}}{\partial p}>0$ and $\frac{\partial \Delta V_{p}}{\partial p}<0$ hold, respectively. Similarly, denote $\mathcal{B}^{+}$, and $\mathcal{B}^{-}$as the set of values of $(p, B)$ where $\frac{\partial \Delta V_{p}}{\partial B}>0$ and $\frac{\partial \Delta V_{p}}{\partial B}<0$ hold, respectively. The strategy going forward is to divide the parameter space into two regions, and to show that under the given assumptions $(p, B)$ can only reside in regions that ensures monotonicity of $p^{*}(B ; N, \cdots)$ in $B$.

Lemma 4. (i) On $\mathcal{C}, \mathcal{B}^{-} \subset \mathcal{P}^{+}$and conversely, $\mathcal{P}^{-} \subset \mathcal{B}^{+}$.

(ii) On $\mathcal{C}^{c}, \mathcal{B}^{+} \subset \mathcal{P}^{-}$and conversely, $\mathcal{P}^{+} \subset \mathcal{B}^{-}$.

Proof. $(i)$ On $\mathcal{C}, f(U)-f(D)>0$. Note, after a little rearrangement of (17) and (18), that

$$
\frac{\partial \Delta V_{p}}{\partial p}>0 \Longleftrightarrow f(U)-f(D)>\frac{N}{2}\left(\left(\frac{1}{2}-\epsilon\right) f^{\prime}(U)+\left(\frac{1}{2}+\epsilon\right) f^{\prime}(D)\right)
$$

and

$$
\frac{\partial \Delta V_{p}}{\partial B}<0 \Longleftrightarrow f(U)-f(D)>N\left(\left(\frac{1}{2}-\epsilon\right) f^{\prime}(U)+\left(\frac{1}{2}+\epsilon\right) f^{\prime}(D)\right) .
$$

Clearly, (20) implies (19) because $f(U)-f(D)>0$ on $\mathcal{C}$.

(ii) On $\mathcal{C}^{c}, f(U)-f(D)<0$. Note, after a little rearrangement of (17) and (18), that

$$
\frac{\partial \Delta V_{p}}{\partial p}<0 \Longleftrightarrow f(U)-f(D)<\frac{N}{2}\left(\left(\frac{1}{2}-\epsilon\right) f^{\prime}(U)+\left(\frac{1}{2}+\epsilon\right) f^{\prime}(D)\right)
$$

and

$$
\frac{\partial \Delta V_{p}}{\partial B}>0 \Longleftrightarrow f(U)-f(D)<N\left(\left(\frac{1}{2}-\epsilon\right) f^{\prime}(U)+\left(\frac{1}{2}+\epsilon\right) f^{\prime}(D)\right) .
$$

Clearly, (22) implies (21) because $f(U)-f(D)<0$ on $\mathcal{C}^{c}$. 
Lemma 4 essentially tells us which parameter spaces are compatible with one another, and this allows us to exclude regions that do not make sense. The Lemma that follows complements Lemma 4 with an additional criterion.

Lemma 5. In equilibrium, $\left(p^{*}, B\right) \notin \mathcal{P}^{+}$.

Proof. This follows from the second order condition on $V$.

Using the above two Lemmas, we can now show a sufficient condition for the proof of the Proposition.

Lemma 6. It suffices to show that $f^{\prime}(U)+f^{\prime}(D)>0$ on every $\left(p^{*}, B\right)$.

Proof. Note first that to ensure monotonicity, we need $\left(p^{*}, B\right) \in\left(\mathcal{P}^{+} \cap \mathcal{B}^{-}\right) \cup\left(\mathcal{P}^{-} \cap \mathcal{B}^{+}\right)$by virtue of the implicit function theorem. Hence it suffices to show that $\left(p^{*}, B\right) \notin\left(\mathcal{P}^{+} \cap \mathcal{B}^{+}\right) \cup\left(\mathcal{P}^{-} \cap \mathcal{B}^{-}\right)$. And moreover, Lemma 5 further reduces the sufficient condition to showing that $\left(p^{*}, B\right) \notin\left(\mathcal{P}^{-} \cap \mathcal{B}^{-}\right)$, simply because $\mathcal{P}^{+}$cannot contain $\left(p^{*}, B\right)$. On $\mathcal{C}$, this condition is trivially satisfied, since by Lemma $4-(i),\left(\mathcal{P}^{-} \cap \mathcal{B}^{-}\right)=\varnothing$. It thus suffices to show that $\left(p^{*}, B\right) \notin\left(\mathcal{P}^{-} \cap \mathcal{B}^{-}\right) \cap \mathcal{C}^{c}$. Note that this means that the following three must not occur simultaneously:

$$
\begin{gathered}
f(U)-f(D)<0 \\
f(U)-f(D)<\frac{N}{2}\left(\left(\frac{1}{2}-\epsilon\right) f^{\prime}(U)+\left(\frac{1}{2}+\epsilon\right) f^{\prime}(D)\right),
\end{gathered}
$$

and

$$
f(U)-f(D)>N\left(\left(\frac{1}{2}-\epsilon\right) f^{\prime}(U)+\left(\frac{1}{2}+\epsilon\right) f^{\prime}(D)\right) .
$$

But clearly, for this, it is sufficient to show that

$$
\left(\left(\frac{1}{2}-\epsilon\right) f^{\prime}(U)+\left(\frac{1}{2}+\epsilon\right) f^{\prime}(D)\right)>0
$$

because it would contradict

$$
N\left(\left(\frac{1}{2}-\epsilon\right) f^{\prime}(U)+\left(\frac{1}{2}+\epsilon\right) f^{\prime}(D)\right)<f(U)-f(D)<\frac{N}{2}\left(\left(\frac{1}{2}-\epsilon\right) f^{\prime}(U)+\left(\frac{1}{2}+\epsilon\right) f^{\prime}(D)\right) .
$$

Furthermore, since $f^{\prime}(U) \leq 0, f^{\prime}(D) \geq 0$, and $\epsilon \in\left[0, \frac{1}{2}\right]$,

$$
\left(\frac{1}{2}-\epsilon\right) f^{\prime}(U)+\left(\frac{1}{2}+\epsilon\right) f^{\prime}(D) \geq f^{\prime}(U)+f^{\prime}(D)
$$

hence $f^{\prime}(U)+f^{\prime}(D)>0$ implies (23), as required. 
To conclude the proof, we show that the sufficient condition, $f^{\prime}(U)+f^{\prime}(D)>0$, holds under our given assumptions: "single-crossing" property and $\frac{N}{2}>\sigma$.

Lemma 7. Under the given assumptions, $f^{\prime}(U)+f^{\prime}(D)>0$.

Proof. We divide into three cases. (i) When $D<-\sigma$, by single crossing, symmetry, and $\frac{N}{2}>\sigma$, $f^{\prime}(D)>\left|f^{\prime}(U)\right|$, so $f^{\prime}(U)+f^{\prime}(D)>0$ always holds. On the other hand, when $D>-\sigma$, again by the assumptions and IVT, it is straightforward to show that $\exists$ at least one pair ${ }^{33}$ of $(D, U)$, such that $f^{\prime}(U)+f^{\prime}(D)=0$. Denote as $D^{\prime}$, the smallest of such $D^{\prime}$ s. (ii) When $-\sigma \leq D<D^{\prime}$, clearly under current assumptions and by definition, $f^{\prime}\left(D^{\prime}\right)+f^{\prime}\left(D^{\prime}+N\right)>0$. (iii) Finally, regarding the possibility that $D$ is in the remaining region, $D^{\prime} \leq D \leq-B=\left.D(\epsilon)\right|_{\epsilon=\frac{1}{2}}$, we ensure that $p^{*}$ can never be such that $D \in\left[D^{\prime},-B\right]$ by defining $\underline{N}$ as follows, and requiring that $\underline{N}<N<\bar{N}$.

$$
\underline{N}:=\max \left(2 \sigma, \inf _{\tilde{N}}\left\{\tilde{N} \in[2 \sigma, \infty): \Delta V_{p}\left(N, p_{0}(N)\right)<0, \forall N>\tilde{N}\right\}\right),
$$

where

$$
p_{0}(N):=\inf _{p}\left\{p \in\left(\frac{1}{2}, 1\right]: f^{\prime}(U)+f(D)=0\right\} .
$$

This definition ensures that $D \notin\left[D^{\prime},-B\right]$ for any given $N>\underline{N}$, so that $f^{\prime}(U)+f^{\prime}(D)>0$ always holds. The final remaining task is to show that $\underline{N}$ is well-defined, which we do in the following Lemma.

Lemma 8. $p_{0}(N)$, and $\underline{N}$ are well-defined.

Proof. We prove only for $B=0$, extending this to all $B>0$ is straightforward. ${ }^{34}$ We start by noting that for any fixed set of parameters, $\lim _{N \rightarrow \infty} \Delta V_{p}(N, p)=-\infty$, and hence $\exists N_{0}<\infty$ such that $\Delta V_{p}\left(N_{0}, p\right)<0, \forall N \geq N_{0}$. In particular, this holds for any given $D:=-N\left(\frac{1}{2}-\epsilon\right)-B$. We denote this point as $N_{0}(D)$.

Meanwhile, define $g(N, \epsilon):=f^{\prime}(U)+f^{\prime}(D)=f^{\prime}\left(-N\left(\frac{1}{2}-\epsilon\right)-B\right)+f^{\prime}\left(N\left(\frac{1}{2}+\epsilon\right)-B\right)$. For any given $N>2 \sigma$, note that $g(N, 0)>0$ and $g\left(N, \frac{1}{2}\right)<0$, and thus it is always possible to find at least one $\epsilon$ such that $g(N, \epsilon)=0$ for that particular $N$. Let $\epsilon_{0}(N)$ be the infimum (if multiple) of such. Clearly, such an element exists by IVT, and is unique by construction, hence it is well-defined.

\footnotetext{
${ }^{33}$ Of course, $U=D+N$ so specifying $D$ automatically fixes $U$. Moreover, fixing an $N$, one can express $D$ and $U$ as $D(\epsilon)$ and $U(\epsilon)$, from the relationships $D:=-N\left(\frac{1}{2}-\epsilon\right)$ and $U:=N\left(\frac{1}{2}+\epsilon\right)$.

${ }^{34}$ This can be done by first noting that $B>0$ restricts the upper end of $D$. If for a given $N, D^{\prime}(N)>-B$, then the statement holds trivially. If $D^{\prime}(N) \leq-B$, then we can import the same arguments introduced in this proof with $B=0$, keeping in mind that, "eventually", $D^{\prime}(N)>-B$ can be induced by increasing $N$.
} 
Furthermore, $\epsilon_{0}\left(N_{1}\right) \neq \epsilon_{0}\left(N_{2}\right)$ for any $N_{1} \neq N_{2}$, hence $p_{0}(N):=\frac{1}{2}+\epsilon_{0}(N)$ is well-defined, and moreover, invertible. We can then construct $\underline{N}$ as follows. Give that $p_{0}(N)$ is well-defined and invertible, specifying an $N$ specifies a $p_{0}(N)$, which in turn, specifies ${ }^{35}$ a $D^{\prime}:=-N\left(\frac{1}{2}-\epsilon_{0}(N)\right)-B$, and consequently, $N_{0}\left(D^{\prime}\right)$. Let $\underline{\mathrm{N}}=: \inf _{N}\left\{N \in[2 \sigma, \infty): N \geq N_{0}(N)\right\}$. One can easily verify that this satisfies the definition of $\underline{N}$ and therefore, $\underline{\mathrm{N}}$ is well-defined.

Q.E.D.

\section{Proof of Proposition 5}

Proof. (i) This follows from $\frac{\partial V}{\partial N}>0, \forall p \in\left(\frac{1}{2}, 1\right], \forall B \geq 0$, which be shown easily, analogous to the proof of Proposition 1.

(ii) The proof mirrors, and relies on facts we have gathered while proving Proposition 5-(ii). We start by the implicit function theorem and recycle some of the earlier results.

$$
\begin{aligned}
\frac{\partial p^{*}}{\partial N} & =-\frac{\frac{\partial \Delta V_{p}}{\partial N}}{\frac{\partial \Delta V_{p}}{\partial p}} \\
& =-\frac{2 \epsilon(f(U)-f(D))+N\left(\frac{1}{2}-\epsilon\right)\left(\frac{1}{2}+\epsilon\right)\left(f^{\prime}(D)-f^{\prime}(U)\right)}{N\left(2(f(U)-f(D))-N\left(\left(\frac{1}{2}-\epsilon\right) f^{\prime}(U)+\left(\frac{1}{2}+\epsilon\right) f^{\prime}(D)\right)\right)} \\
& =-\frac{\frac{2 \epsilon(f(U)-f(D))}{N}+\left(\frac{1}{2}-\epsilon\right)\left(\frac{1}{2}+\epsilon\right)\left(f^{\prime}(D)-f^{\prime}(U)\right)}{2(f(U)-f(D))-N\left(\left(\frac{1}{2}-\epsilon\right) f^{\prime}(U)+\left(\frac{1}{2}+\epsilon\right) f^{\prime}(D)\right)} .
\end{aligned}
$$

Our task is to sign the above expression as $N$ tends to infinity. Note that the first terms in the numerator and denominator become immaterial, at least towards the purpose of signing $\frac{\partial p^{*}}{\partial N}$ as $N \rightarrow \infty$. Since the term $f^{\prime}(D)-f^{\prime}(U)$ in the numerator is positive in our setup, it suffices to show that $\left(\frac{1}{2}-\epsilon\right) f^{\prime}(U)+\left(\frac{1}{2}+\epsilon\right) f^{\prime}(D)$ is "eventually" positive as $N$ increases. This, in turn, is ensured by showing that $f^{\prime}(U)+f^{\prime}(D)>0$ "eventually", which has been done in the proof of Proposition 5. This establishes that $\lim _{N \rightarrow \infty} \frac{\partial p^{*}}{\partial N}=0^{+}$, and therefore $\exists \tilde{N}<\infty$ such that $\frac{\partial p^{*}}{\partial N}>0$ whenever $N>\tilde{N}$. Q.E.D.

\section{Proof of Proposition 6}

Proof. (i) We show this with two Lemmas.

Lemma 9. Fix $N \leq \bar{N}$. For any $B,\left.\Delta V_{p}\right|_{\left\{p=\frac{1}{2}+\frac{B}{N}\right\}}>0$.

\footnotetext{
${ }^{35}$ Note that because $\epsilon_{0}$ is defined as an infimum (or minimum), $D=-N\left(\frac{1}{2}-\epsilon_{0}\right)-B$ is, in fact, $D^{\prime}$.
} 
Proof. When $\epsilon=\frac{B}{N}$,

$$
\begin{aligned}
\left.\frac{\partial V(N, p ; B)}{\partial p}\right|_{\left\{p=\frac{B}{N}\right\}} & =\left(F\left(\frac{1}{2} N \mid 0\right)-F\left(-\frac{1}{2} N \mid 0\right)\right) \\
& -N\left(\left(\frac{1}{2}-\frac{B}{N}\right) F^{\prime}\left(\frac{1}{2} N \mid 0\right)+\left(\frac{1}{2}+\frac{B}{N}\right) F^{\prime}\left(-\frac{1}{2} N \mid 0\right)\right) \\
& =\left(F\left(\frac{1}{2} N \mid 0\right)-F\left(-\frac{1}{2} N \mid 0\right)\right)-N \cdot F^{\prime}\left(\frac{1}{2} N\right) \\
& =\int_{-\frac{1}{2} N}^{\frac{1}{2} N}\left(F^{\prime}(x \mid 0)-F^{\prime}\left(\frac{1}{2} N \mid 0\right)\right) d x \\
& >0
\end{aligned}
$$

where the last inequality follows from uni-modality.

Lemma 10. $\exists N_{0}$ such that $\left.\Delta V_{p}\right|_{\left\{B=-\frac{N}{2}, p=\frac{1}{2}\right\}}<0$ whenever $N>N_{0}$.

Proof.

$$
\begin{aligned}
\left.\Delta V_{p}\right|_{\left\{B=-\frac{N}{2}, p=\frac{1}{2}\right\}} & =F(N \mid 0)-F(0 \mid 0)-N\left(\frac{F^{\prime}(N \mid 0)+F^{\prime}(0 \mid 0)}{2}\right) \\
& <\frac{1}{2}-\frac{N}{2} F^{\prime}(0 \mid 0) \longrightarrow-\infty \text { as } N \rightarrow \infty
\end{aligned}
$$

whence the claim follows from standard argument by contradiction.

Now let $\hat{N}$ be the smallest (or infimum) of all the $N_{0}$ that satisfy Lemma 10. Fix any $N>N_{0}$. Since Lemma 9 holds for all $B$, apply the Lemma for $B=-\frac{N}{2}<0$, (whence $p:=\frac{1}{2}+\frac{B}{N}=\frac{1}{2}-\frac{1}{2}=0<\frac{1}{2}$ ) obtaining $\left.\Delta V_{p}\right|_{\left\{B=-\frac{N}{2}, p=0\right\}}>0$. This, combined with Lemma 10 allows us to use the IVT, viewing $\Delta V_{p}$ as a single-valued function on $p$ and fixing all other parameters, whereby it follows that $\exists p^{*} \in\left(0, \frac{1}{2}\right)$ such that $\left.\Delta V\right|_{\left\{p=p^{*}\right\}}=0$.

(ii) The proof of Proposition 5-(ii) extends effortlessly to this case $(B<0)$. Q.E.D. 